\title{
FLUID DISINTEGRATION STUDIES IN A SPECIALIZED SHOCK TUBE
}

\author{
I. Stotz, G. Lamanna, and B. Weigand \\ Institut für Thermodynamik der Luft- und Raumfahrt (ITLR) \\ Universität Stuttgart \\ Pfaffenwaldring 31, Stuttgart 70569, Germany
}

This paper describes a double-diaphragm shock tube, designed for the experimental investigation of fundamental processes related to fluid disintegration and mixing at elevated pressures and temperatures, representative for realistic engine conditions. Special features of the shock tube include a variable-area driver section to compensate for shock attenuation and boundary layer effects. In discussing the shock tube operational envelope, particular emphasis is given to the requirements to be fulfilled to attain reproducible and accurate experimental data and to capture the most relevant features of subcritical and supercritical mixing behavior. A review of potential thermodynamic transitions for near-critical jet disintegration phenomena is presented and the main features of each disintegration mode are discussed on the basis of the shock tube experiments.

\section{INTRODUCTION}

Thorough understanding of the injection, disintegration, and combustion processes of liquid jets is of particular importance with respect to many propulsion systems, such as rocket engines, gas turbines, (sc)ramjets and direct injection engines. In applications where high heat loads are involved, which is always the case in high-speed flight or in combustion chambers, the fuel is often used to cool the structures and is, therefore, preheated before injection and combustion. Intense fuel preheating can transfer the fuel into a superheated or supercritical state prior to injection, which can influence the breakup and mixing process in the combustion chamber. A qualitative survey of the possible thermodynamic transformations is presented in Fig. 1. The starting point refers to the stagnation conditions (i.e., reservoir conditions) and the final point refers to the ambient conditions in the combustion chamber. Note that the selected thermodynamic processes are always related to current (or potential) applications in

This is an Open Access article distributed under the terms of the Creative Commons Attribution-Noncommercial License 3.0, which permits unrestricted use, distribution, and reproduction in any noncommercial medium, provided the original work is properly cited. 


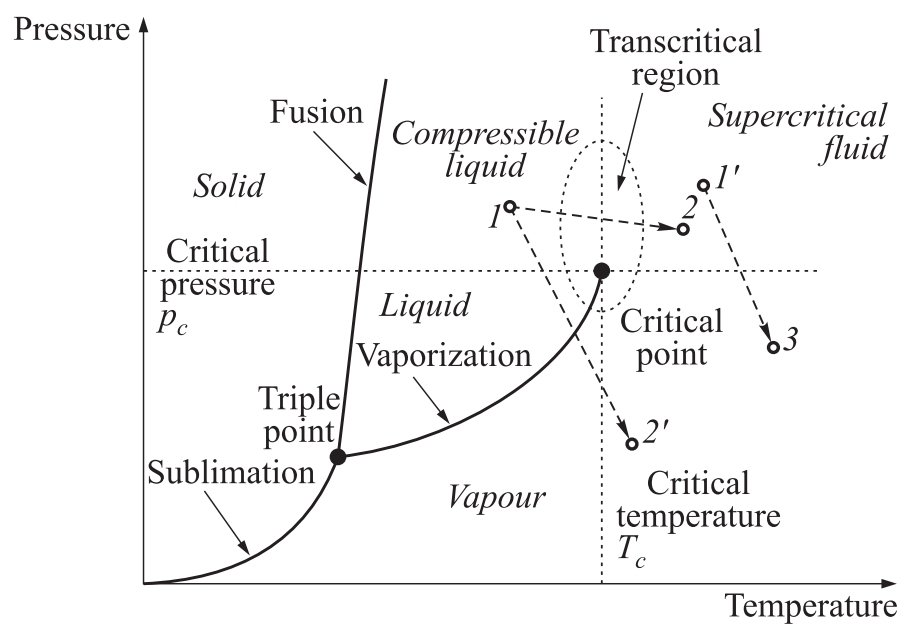

Figure 1 Qualitative thermodynamic transitions of jet disintegration at trans- and supercritical ambient conditions

the aerospace and automotive industry. The state of the injected fuel is characterized through two reference parameters: the reduced temperature $T_{r}=T_{\mathrm{inj}} / T_{c}$ and the reduced pressure $p_{r}=p_{\mathrm{chm}} / p_{c}$, respectively. The subscript " $c$ " refers to the critical condition.

Transformation 1 to 2 is frequently encountered in liquid propellant rocket technologies, where liquid fuel at supercritical pressure but subcritical temperature is injected in an environment, where both pressure and temperature exceed the jet critical condition. In the following sections, this disintegration mode is denoted as the transcritical disintegration regime. In this regime, the thermodynamic properties of the fuel may strongly influence the breakup process. As an example, the rapid increase of specific heat at the critical point implies that much more thermal energy is needed to heat up the transcritical jet, thus drastically affecting the heat transfer process between the jet and the ambient gas. Further anomalous variations of fluid volumetric and thermal properties in the proximity of the critical point, such as liquid-like densities, vanishing of surface tension and enthalpy of vaporization, enhanced values of thermal conductivity, viscosity, and mass diffusivity, all contribute to alter the driving forces and the dynamics of jet disintegration at high pressure. Surface tension, for example, is expected to play a dominant role only in the initial phase of jet breakup. As soon as, locally, the critical point of the fluid is reached, turbulence and shear-layer instabilities as well as an additional transport mechanism due to thermal diffusion effects (e. g., Soret and Dufour effects) become of pronounced importance in this regime and govern the separation of ligaments from the jet $[1-5]$. 
Transformation 1 to $2^{\prime}$ is of significant interest for the potential use of liquid hydrocarbon fuels in propulsion systems for hypersonic flight applications. In these applications, the hydrocarbon fuel could be used as coolant to handle the high heat loads associated with hypersonic flights, and thus might subsequently be injected as superheated liquid. Several tests have shown that the injection of preheated liquid jets at supercritical pressure may result in the inception of flash-boiling. This disintegration mode, termed near-critical flashing regime, features many characteristics similar to the "flash" atomization process at low pressure conditions. In this case, liquid fragmentation is due to a phase transition process within the jet. Bubble nucleation and accompanying growth are responsible for rapid evaporation within the liquid jet and its subsequent shattering. In the proximity of the critical point, though the diminished surface tension results in a drastic reduction of the energy barrier to nucleation, and hence, in the earlier onset of nucleate boiling. Furthermore, thermodynamic and transport anomalies near the critical point also impact the mass and heat transfer between the injectant fluid and supersonic stream. In [6], it is shown, for example, that the structure and penetration of a near-critical superheated jet not only depends upon the initial degree of superheat, but is also strongly interlinked with the heat transfer rate between the jet and the main flow.

If the fuel is used for the thermal management of the airframe and combustor, the fuel operating pressure and temperature may exceed the critical condition and induce the transition to supercritical state. In this hypothesis, an additional thermodynamic transition should be considered (supercritical regime), qualitatively represented by the transformation from state $1^{\prime}$ to 3 . The injection of fuels at supercritical conditions is thought to be favourable for promoting rapid mixing and ignition, as it should be most similar to gas-phase injection. Still, depending on the injection and chamber conditions, it cannot be excluded that, during the expansion, the pressurized fuel may become locally supersaturated and initiate condensation through homogeneous and/or heterogeneous nucleation. Similarly to the near-critical flashing regime, also for the transformation $1^{\prime}$ to 3 , vanishing of surface tension, reduced heat transfer and mass diffusion due to thermodiffusional effects have a strong impact on the onset of nucleation, dispersed phase properties, composition, and structure of two-phase jet. This analysis points to a need for an improved fundamental understanding of the physics of supercritical fluid injection, nonequilibrium homogeneous condensation, and fuel/oxidant mixing in highly compressible (i. e., near-critical) flows in order to provide design criteria for the successful development of scramjet propulsion systems.

From this brief review, it appears clearly that the term "near-critical" disintegration includes de facto a number of complex and diversified nonequilibrium thermodynamic processes, which include transport anomalies due to Soret and Dufour effects, additional turbulent dissipation with significant contributions from the cross-term proportional to molecular and thermal diffusion, near-critical 
homogeneous condensation, and nucleate boiling. The inception and relevance of these processes is strongly dependent upon the local thermodynamic conditions. It is, therefore, of paramount importance to perform the experiments under realistic combustor conditions and to maintain the injection and test-chamber conditions constant in time and uniform in space during jet disintegration experiments. Unintended changes in the thermodynamic state, in fact, may locally promote or inhibit the onset of thermodynamic and transport anomalies, thus impacting greatly the jet disintegration and mixing process.

This paper discusses a specialized shock tube designed to investigate such processes via simplified and systematic experiments under actual combustor conditions. The ITLR shock tube is equipped with a variable-area driver section to compensate for boundary layer effects and to guarantee uniformity of thermodynamic conditions in the measurement section. A detailed description of the construction and features of the shock tube is presented in [7]. The primary objective of the present study is to demonstrate the feasibility of near-critical jet disintegration studies in the ITLR shock tube. For this purpose, a precise rationale is pursued. Section 2 examines the main features of shock tube flow and its limitations in practical applications. Section 3 briefly discusses the main design features of the ITLR shock tube and injection system, while section 4 provides a detailed analysis of the shock tube performance in terms of uniformity of test conditions, uncertainty in the measurement of shock speed, and determination of reflected shock properties. Section 5 concisely describes the optical setup for high-speed Schlieren and Mie imaging together with the postprocessing algorithm. Finally, in section 6, the feasibility of near-critical jet disintegration experiments in the ITLR specialized shock tube is demonstrated by comparing own experimental findings with literature data.

\section{SHOCK TUBE THEORY}

\subsection{Shock Tube Flow}

The most simple shock tube configuration shown in Fig. $2 a$ consists of two cylindrical tubes with equal diameters separated by a diaphragm. The high-pressure part of the shock tube is called driver (section) and contains the driver gas (state 4). The latter is pressurized to a level higher than the test gas (state 1), which is filled into the low-pressure part, called the driven section. Upon rupture of the diaphragm, a shock wave builds up and propagates down the driven section with the (supersonic) velocity $u_{s}$ (shock Mach number $\mathrm{Ma}_{s}>1$ ). At the same time, an expansion fan (centered at $x=0, t=0$ ) travels in the opposite direction into the driver section. Neglecting all viscous losses and assuming instantaneous (local) thermodynamic equilibrium and ideal diaphragm behavior 


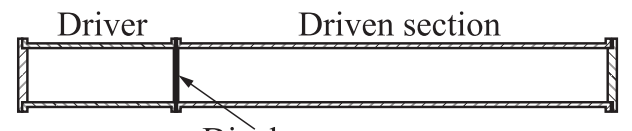

Diaphragm

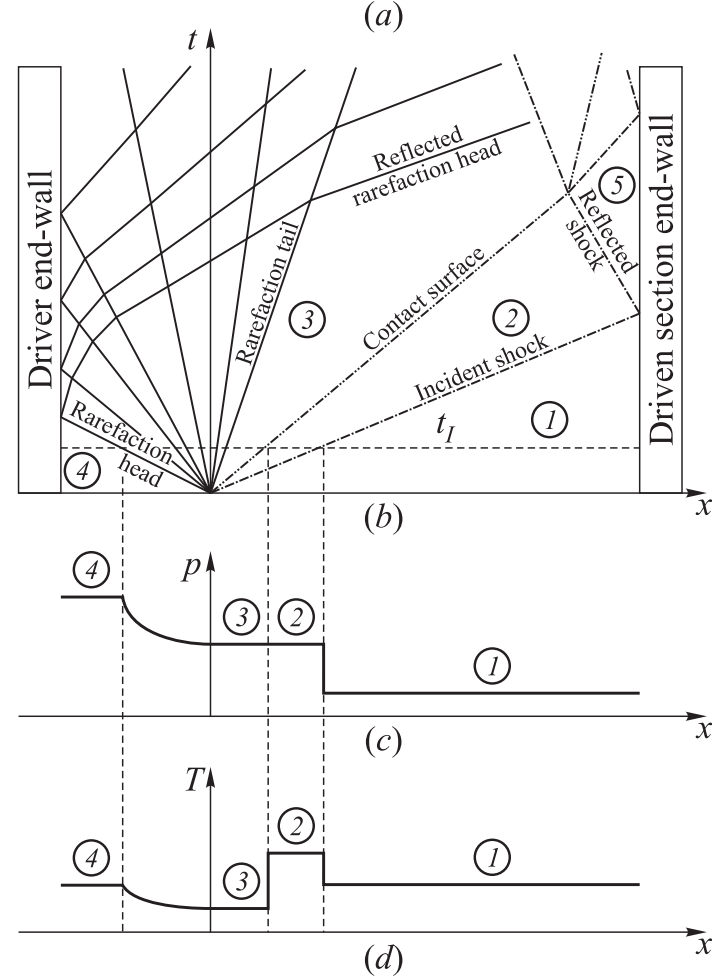

Figure 2 Gas dynamics and shock wave propagation in an ideal shock tube: $(a)$ simple shock tube configuration; (b) $x, t$-diagram (wave diagram); (c) pressure distribution $\left(t=t_{\mathrm{I}}\right) ;$ and $(d)$ temperature distribution $\left(t=t_{\mathrm{I}}\right)$

(i. e., infinitesimal thickness, complete planarity before rupture, instantaneous opening time, no influence on the successive flow), the processes can be pictured as shown in Fig. $2 b$.

Once the incident shock wave hits the rigid end-wall of the driven section, it is reflected and travels in the opposite direction (shock velocity $u_{r}$ ). It further increases the pressure and temperature of the test gas and brings it to rest (state 5 ). State 5 provides quiescent steady-state conditions for a specific test time $\Delta t_{5}$ (usually, in the range of a few milliseconds). In many shock tube applications, reflections of normal shock waves from flat (rigid) walls play an 
important role, especially when the stagnation properties of the gas behind a reflected shock are of relevance. Therefore, the understanding of the processes involved is indispensable. The reflected properties (index 5) can be derived when the one-dimensional (1D) normal shock equations are solved across the incident and the reflected shock wave. With these considerations, the following shock relations, expressing the reflected shock conditions solely as functions of the (experimentally measured) incident shock Mach number $\mathrm{Ma}_{s}$, yield:

$$
\begin{aligned}
\frac{p_{5}}{p_{1}} & =\frac{2 \gamma \mathrm{Ma}_{s}^{2}-(\gamma-1)}{\gamma+1} \frac{(3 \gamma-1) \mathrm{Ma}_{s}^{2}-2(\gamma-1)}{(\gamma-1) \mathrm{Ma}_{s}^{2}+2} \\
\frac{T_{5}}{T_{1}} & =\frac{\left(2(\gamma-1) \mathrm{Ma}_{s}^{2}+(3-\gamma)\right)\left((3 \gamma-1) \mathrm{Ma}_{s}^{2}-2(\gamma-1)\right)}{(\gamma+1)^{2} \mathrm{Ma}_{s}^{2}} \\
\frac{\rho_{5}}{\rho_{1}} & =\frac{(\gamma+1) \mathrm{Ma}_{s}^{2}\left(2 \gamma \mathrm{Ma}_{s}^{2}-(\gamma-1)\right)}{\left((\gamma-1) \mathrm{Ma}_{s}^{2}+2\right)\left(2(\gamma-1) \mathrm{Ma}_{s}^{2}+(3-\gamma)\right)}
\end{aligned}
$$

These are the essential equations for the computation of the test conditions in shock tube experimentation. They enable the direct calculation of pressure, temperature, and density behind the reflected shock wave, if the initial filling conditions in the driven section 1 are known and the incident shock speed $u_{s}$ (the shock Mach number $\mathrm{Ma}_{\mathrm{s}}$, respectively) is measured.

Isentropic postshock changes of state can be calculated applying the isentropic relation

$$
\frac{p}{\rho^{\gamma}}=\text { const }
$$

to the flow. This equation is particularly useful in the calculation of real shock tube flows where pressure, temperature, and density do not stay perfectly constant on their immediate postshock values due to nonideal effects (see subsection 2.2) or where changes due to rarefaction waves, which induce isentropic changes of state, are involved.

\subsection{Nonideal Effects in Shock Tubes due to Boundary Layer Growth}

Viscosity and real-gas effects, nonideal diaphragm behavior, and wall heat transfer can have a severe influence on shock tube performance and cannot, therefore, usually be neglected. Especially viscous shock attenuation due to boundary layer growth can considerably alter the test conditions, as it can cause increasing pressures and temperatures over time. In this section, a phenomenological explanation of this effect is provided and its implications on the shock tube flow are discussed. 
The pioneering work of [8] provides a simple but effective approach for modeling the interaction between the boundary layer and the travelling shock wave. To date, it still constitutes the basis for many shock tube boundary layer and shock attenuation models (see, e.g., $[9,10]$. The core assumptions of these models are presented hereafter. As the incident shock wave propagates along the driven section, a boundary layer builds up behind it because of wall viscous effects. The boundary layer induces a cross velocity in the main flow, which can be treated as a mass sink. The cross flow, i. e., the entrainment into the boundary layer, induces rarefaction waves in the core flow. These (complex) waves can be treated as axial density variations (as a function of $x$ and $t$ ), which emanate from mass sinks distributed uniformly over the tube cross section. Eventually, the waves lead to the attenuation of the incident shock wave and, hence, to the deviation of the test gas properties between the shock wave and the contact surface 2 from those calculated for the ideal inviscid case. Typically, shock attenuation is of the order of $1-2 \% / \mathrm{m}$ for low-pressure shock tubes, whereas attenuations of $2-4 \% / \mathrm{m}$ are common in high-pressure applications. Here, $70 \%$ or more of the total shock attenuation is caused by viscous effects in the boundary layer [11], the remainder is attributed to nonideal shock formation due to nonideal diaphragm rupture. Besides the attenuation of the incident shock wave, the reflected shock propagates into spatially nonuniform test gas, as the flow velocity $u_{2}$ changes continuously and, thus, the fluid experiences a temperature and pressure gradient.

Considering these nonideal effects occurring in real shock-tube flows, the ideal picture given in Fig. 2 significantly changes. The incident shock wave deviates from its ideal constant velocity and is slowed down as it propagates along the shock tube. Consequently, the shock strength is attenuated and, thus, the gas properties behind the shock wave vary along the shock tube axis: the further upstream, the higher the postshock values of pressure, temperature, and density. After being reflected from the end-wall, the shock propagates into a flow with spatially inhomogeneous properties and interacts with the boundary layer of the incident shock wave. Therefore, the strength of the reflected shock only "matches" the flow right at the end-wall. Further upstream, the kinetic energy of the oncoming flow is higher as the latter has been compressed by a stronger incident shock. This leads to a flow towards the end-wall and results in pressures and temperatures behind the reflected shock wave increasing with time.

\subsection{Compensation of Shock Attenuation}

A possibility to compensate for shock attenuation effects is to use a driver section of varying cross-sectional area. The idea behind this concept is to take advantage of the rarefaction waves, which are being reflected at the (slightly) inclined side walls of the driver tube, to influence the flow-field behind the reflected shock 
Constant-area:

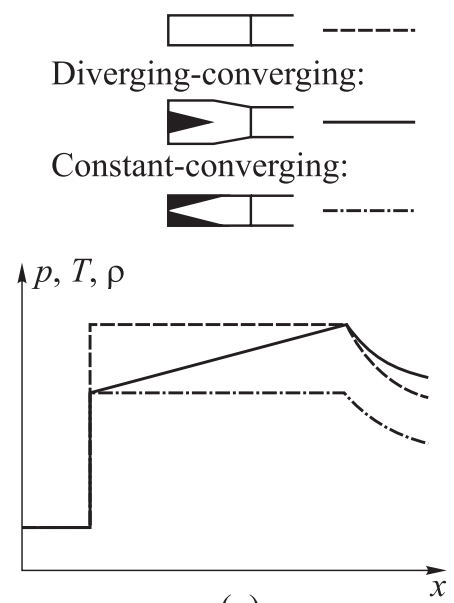

(a)

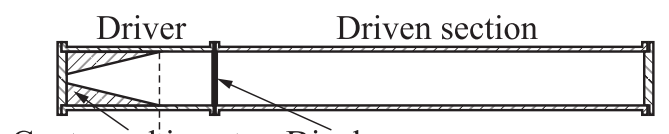

Contouredinsert Diaphragm

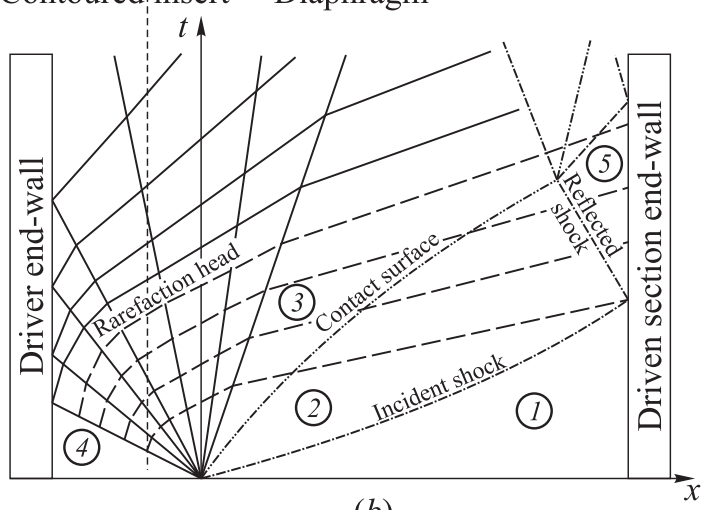

(b)

Figure 3 Influence of the inner contour of the driver section on the flowfield of a shock tube: (a) variation of the conditions in the postshock region with varying driver cross sections; and (b) wave diagram of a shock tube with a constant-convergent driver cross section

wave. Upon reflection, these waves propagate downstream towards the test section, as illustrated graphically in Fig. $3 b$. As soon as these waves reach the region behind the reflected shock, they isentropically compensate the local pressure and temperature rise, thus counterbalancing the boundary layer effects and reestablishing uniform chamber conditions. Basically, the length of the insert determines the timing of the arrival of the reflected expansion fan at the test location, while the rate of driver area contraction determines its strength. The principle feasibility of this method was first demonstrated by Dumitrescu [12], who suggested the application of a driver section with a constant area followed by a gradual area contraction. The beauty of this concept is its simplicity, as an existing facility can be easily upgraded by simply adding an insert or liner with a contour appropriate for the corresponding shock Mach number. This approach does not result in a constant-strength shock wave. However, by counterbalancing the local pressure/temperature increase, it is feasible to produce temporally constant postshock conditions (i. e., pressure, temperature, density), which is the objective of most applications. The variation of the postshock parameters together with the corresponding driver shape is shown in Fig. $3 a$, while the wave diagram of a shock tube with a driver with a gradual area reduction is illustrated in Fig. $3 b$. As can be seen, such a facility is virtually free of shock attenuation. 
The reduction of the driver cross section by employing an appropriately shaped insert has some significant advantages. An existing shock tube can be upgraded with rather low cost and effort and without any major changes to the original facility. The adaptation to different test conditions $\left(\mathrm{Ma}_{s}\right)$ can be easily realized by simply exchanging the inserts. If a set of driver inserts is allocated, a broad range of attenuation free test conditions can be achieved.

\section{TEST FACILITY DESIGN}

\subsection{Shock Tube}

The essential parts of a shock tube are the driver and driven sections. In this particular application, a buffer section is additionally placed between the driver and the driven section. Furthermore, a dump tank is usually applied to provide extra volume in order to reduce the gas pressure and to collect the test gases. All parts of the shock tube are made of stainless steel. All these components are mounted on support frames, which in turn are attached to the laboratory floor via shock absorbers. A schematic drawing of the ITLR shock tube is shown in Fig. 4. The 3.0-meter long driver section has a circular cross section with an internal diameter of $72 \mathrm{~mm}$. A 60-millimeter long buffer connects the driver to the driven section and simultaneously serves as the mounting device for the diaphragms. The driven section has an overall length of $9.36 \mathrm{~m}$. Its major part is also round $(72 \mathrm{~mm})$, while the last meter has a square cross section $(50 \times 50 \mathrm{~mm})$. The transition from the circular to the square part is realized by the introduction of a skimmer which ensures a semidirect connection between both parts.

As previously described, viscous effects influence shock tube performance and test conditions. Preliminary experimental and numerical studies $[7,13]$ showed that, due to boundary layer effects, an increase in pressure and temperature can be observed in the postshock region. In order to compensate for these effects, the shock tube was equipped with a variable-area driver section [7]. A conical variable-slope liner insert is used to produce the continuous driver area reduction (Fig. 5). The contour design and optimization of the inserts were conducted using the quasi-1D code L1d $[14,15]$. The final insert design consists of several single elements, which are bolted together via fine pitch threads. As shown in Fig. 5, the contoured insert is closed by a (double) blind flange, which is equipped with several threaded sockets and ports to mount the rig to the driver section and to allow for evacuation, filling, and venting of the driver chamber. The test section has a square cross section $(50 \times 50 \mathrm{~mm})$. This is necessary if (observation) windows are to be mounted flush with the chamber walls without any recessing edges, thus enabling undisturbed shock propagation. The transition to the test chamber is achieved by the skimmer, which allows for a contactless semidirect 


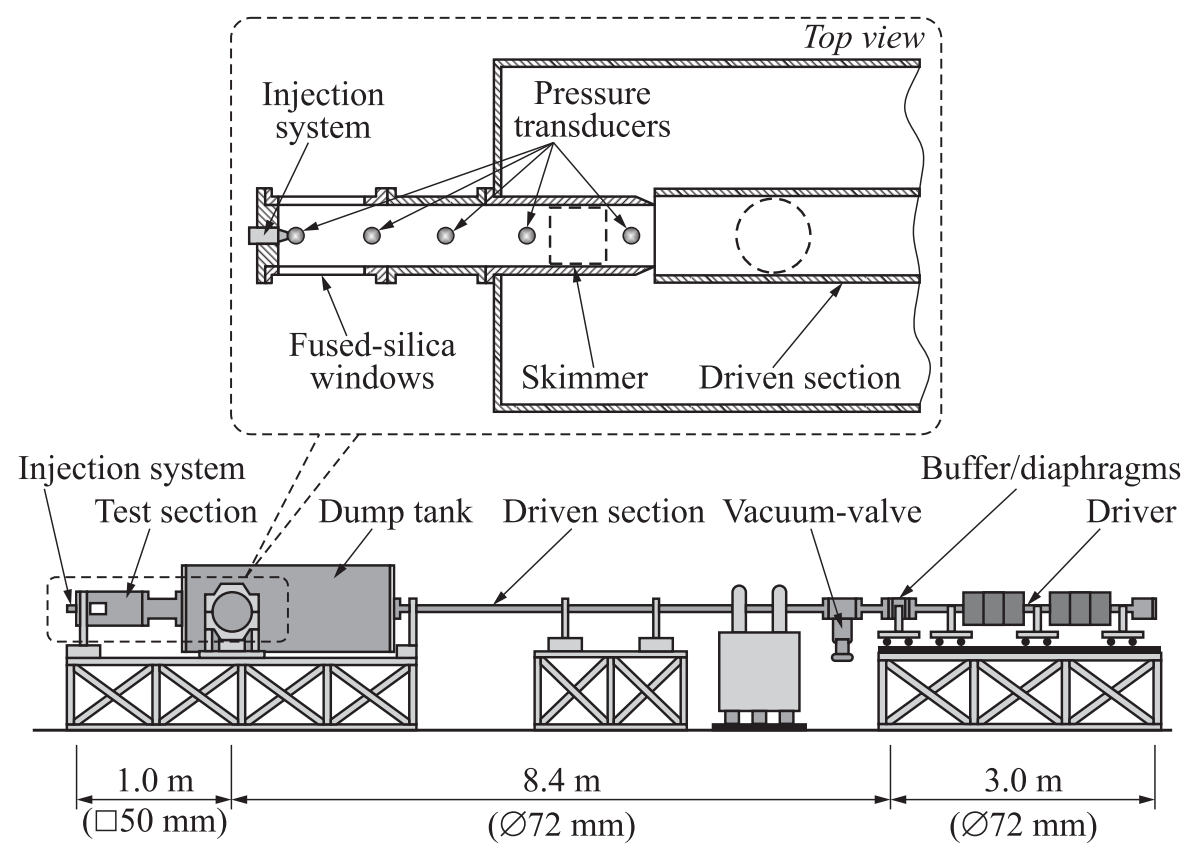

Figure 4 Sketch of the double-diaphragm shock tube

bridging of the round driver tube to the square test chamber without observable losses in shock strength. The functional principle is pretty straightforward: the circular incident shock wave impacts on the (sharp) skimmer tip and a square portion is cut out and propagates further into the test chamber, while the remaining part is fed in the dump tank. The idea of utilizing a skimmer was first proposed by Schardin [16, 17], who employed a skimmer to visualize transient gas dynamic processes in a shock tube. The effects of the skimmer on the

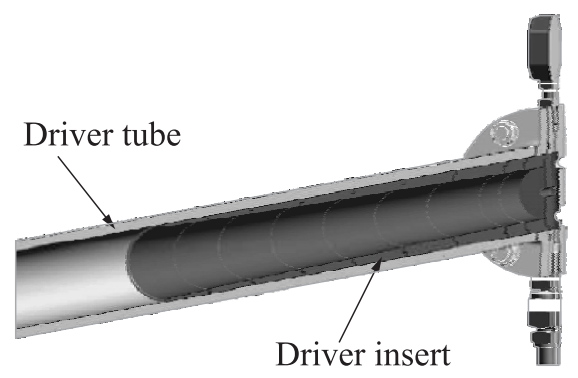

Figure 5 Detailed view of the driver section showing the contoured liner insert 


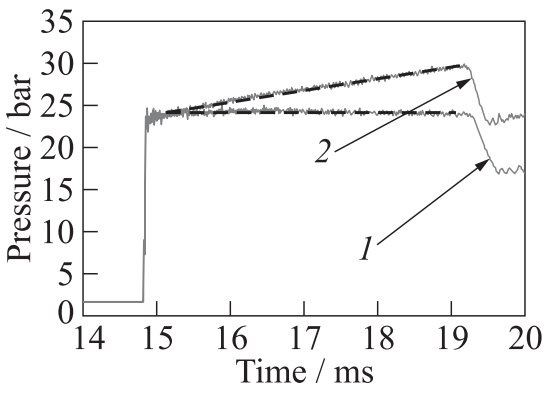

(a)

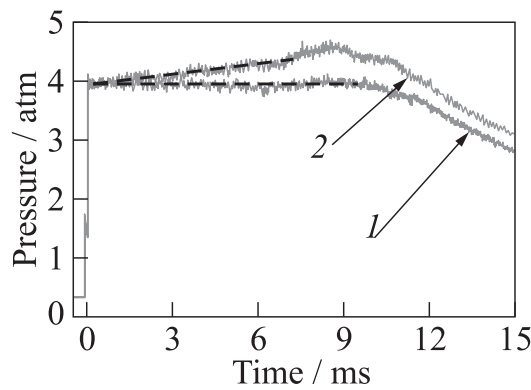

(b)

Figure 6 Experimental pressure traces with (1) and without (2) driver insert from two different facilities. The dashed lines indicate very uniform test conditions when using a driver insert and a linear pressure increase without a driver insert: (a) DDST at the Institute of Aerospace Thermodynamics — ITLR (Universität Stuttgart) [7]; and (b) HPST at the High Temperature Gasdynamics Laboratory (Stanford University) [18]

flow conditions in the test section have been analyzed in detail in [7]. Briefly, it turned out that as the reflected shock wave leaves the skimmer and propagates into the circular tube (i. e., sudden widening of cross section), an expansion fan forms and travels back into the test section. This effect is clearly visible in the pressure recordings, shown in Fig. 6, and results in a shortening of total test time. Neither the skimmer nor the dump tank induce any disturbances on the pressure recordings, which remain constant and uniform in the test section during test time. The test section has a length of $191.5 \mathrm{~mm}$ and allows for the application of windows on all four side walls, thus providing good optical access to the region of interest. The flush-mounted sidewall windows (40 mm thick in order to withstand high test pressures) cover the full height of the duct over the length of $60 \mathrm{~mm}$. The 10-millimeter wide and 60-millimeter long top and bottom windows $(40 \mathrm{~mm})$ can be used to apply laser diagnostics, i. e., to couple a laser beam or sheet into the test chamber.

For the precise calculation of the test conditions, accurate determination of the shock velocity and Mach number is needed. For this purpose, the last meter of the shock tube is equipped with pressure sensors used to detect the shock waves and to record pressure histories. The sensors (fast-response piezoelectric pressure transducers, Kistler 603B) are flush-mounted with the shock tube wall. In order to avoid incorrect readings, they are shielded against heat transfer with a thin layer of red RTV (Room Temperature Vulcanizing) silicone. To reduce the errors involved in the calculation of the shock speed, five ports, which are used to determine the shock Mach number, are spread over the last $845 \mathrm{~mm}$ of the facility. The shock tube closes with an end flange, which also holds the injection system used for the fluid disintegration studies. 


\subsection{Injection System}

In order to be suitable for shock tube applications, both the injector and its periphery have to meet some limiting design requirements. A first prerequisite is the use of a fast-response injector, characterized by short transient times for attaining steady-state injection. A second requirement is the possibility of varying the injection conditions (e.g., pressure and temperature) independently and over a wide range, representative for the operating conditions of modern propulsion systems. Finally, the injection system has to be capable of withstanding pressure and temperature strains and has to be vacuum-sealable. The fuel can be directly heated within the injector itself, so that the target fuel temperature is already reached at the beginning of the injection event. For controlling the fuel temperature, two compact high-performance tubular cartridge heaters were placed around the injection system (Fig. 7). The first one, which is integrated in a stainless steel housing, is used to heat up the fuel in the nozzle to the target temperature, while the second is wrapped around the injector body in order to ensure homogenous fuel temperatures.

The injector is mounted flush with the end-wall of the shock tube in such a way that the liquid fuel is injected perpendicularly to the end-wall into the shocked gas behind the reflected wave as shown in Fig. 7. Two copper gaskets ensured vacuum and pressure sealing of the injector which was attached to the end flange by means of a clamping claw. The end flange was water cooled because of its direct contact with the test chamber and because of the epoxy-

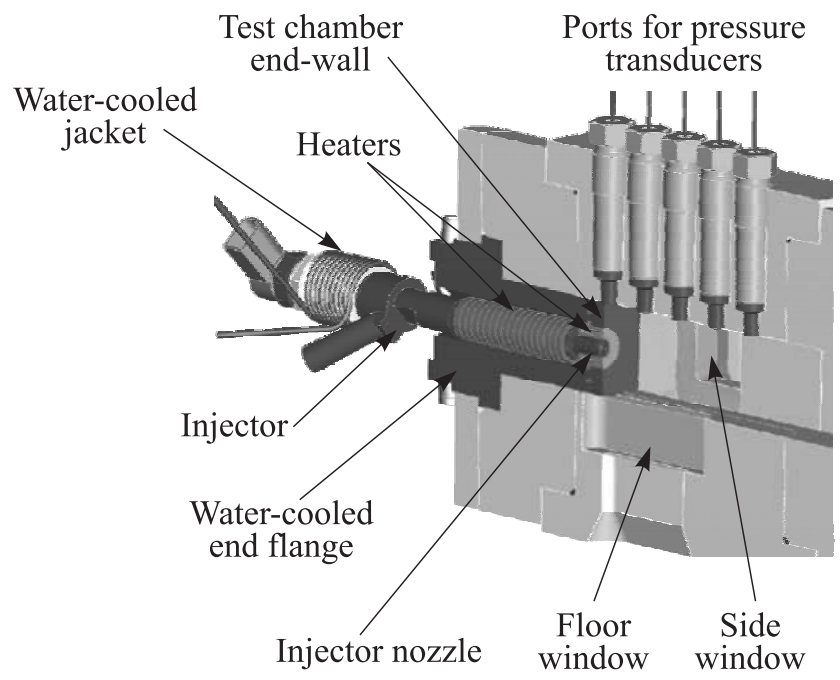

Figure 7 Detailed view of the test chamber equipped with the fuel injector 
embedded windows, which cannot withstand high temperatures. The heaters were electronically regulated by PID controllers. Fuel temperatures of $600 \mathrm{~K}$ and above could be achieved with the present system. An adaption of the standard injector was necessary to avoid damaging its electrical components. The basic idea was to add an additional cooling system to the rear part of the injector, which required the elongation of the injector. An additional water-cooled jacket has been designed to cool the rear part of the injector to keep the temperature sensitive electrical components at moderate temperatures. Due to that, the inner casing and the steering needle, which controls the opening mechanism of the injector, had to be redesigned and extended.

As liquid supply and pressurization system, a Bosch CP-3 high-pressure pump was employed. Fuel injection pressures could be varied between 100 and 1400 bar. The injection was controlled by an electronic control unit which regulated all injection parameters such as the injection pressure, the number of injections, and the duration of the injection process. Typical injection times ranged from a couple of hundreds of microseconds up to about $6 \mathrm{~ms}$.

\section{PROCEDURE, PERFORMANCE, AND TEST CONDITIONS}

\subsection{Procedure}

Before a test was conducted, the shock tube was evacuated to pressures smaller than $10 \mathrm{~Pa}$. A Leybold Trivac D8B rotary vane pump with a volume rate of $8.5 \mathrm{~m}^{3} / \mathrm{h}$ was used to evacuate the driver and buffer sections. The dump tank and driven section were pumped down with an BOC Edwards E2M80 vacuum pump (throughput $80 \mathrm{~m}^{3} / \mathrm{h}$ ). The vacuum pressures were controlled using Leybold Thermovac TR201 and TR211 vacuum gauges with a Leybold Combivac CM31 control unit.

The shock tube filling operations were fully automated. For this purpose, Bürkert (Type 0255) solenoid valves were installed in the gas filling lines. A LabVIEW ${ }^{\mathrm{TM}}$-based programme has been developed to control the filling procedure, based on the input signals of three pressure gauges (Keller PA-21 for buffer and driver, Keller PAA-33X for the driven section). The test gas temperature was monitored with a PT100 resistance thermometer sitting in a side-flange of the dump tank. The experiment was started by venting the buffer section using a Parker Serie 9 valve, which was controlled by a solenoid valve controller (IOTA ONE).

The rapid pressure rise in the buffer caused by the rupture of the first diaphragm was detected by a transient pressure transducer (Kistler 701A). This manually triggered transducer was also used to gauge the diaphragm burst pressure, especially when testing new diaphragms. Additionally, the shock tube 
recoil was detected by a light barrier mounted at the driver section. Both signals were transmitted to a Labsmith (LC880) trigger unit, which activated the pressure transducers mounted along the shock tube. This precaution was necessary to keep the operating time of the piezoelectric transducers as short as possible, in order to avoid natural drift. All other components (cameras, light sources, injection system, etc.) were triggered from one of the downstream pressure transducers. The entire timing of the experiments was controlled by the Labsmith trigger unit.

Transient pressure traces were monitored with fast-response piezoelectric pressure transducers (Kistler 603B) and corresponding charge amplifiers (Kistler 5007 and 5011) and recorded by a Nicolet transient recorder (Team 500) with a 1-megahertz sample rate. These transducers were flush-mounted in 5 ports upstream of the shock tube end-wall and were used to monitor the pressure behind the incident and reflected shock waves. The time delay between shock detection at different transducer locations was subsequently utilized to determine shock wave velocities and Mach numbers.

\subsection{Shock Tube Characterization and Performance}

Satisfactory results could be obtained using analytical and numerical fluid dynamic approaches to layout such a facility [7, 18]. Two sample results from two different facilities (DDST at ITLR - Universität Stuttgart and HPST at the High Temperature Gasdynamics Laboratory — Stanford University) were shown above in Fig. 6. In both cases, the experiments were performed with and without a driver insert at otherwise similar initial conditions (similar filling pressures and gases and thus shock Mach numbers). As can be seen, the pressure rise behind the reflected shock wave could be eliminated almost completely and very uniform test conditions, exhibiting only very minor fluctuations, were obtained in both facilities.

Moreover, for a given target Mach number $\mathrm{Ma}_{s}$ (temperature $T_{5}$, respectively), the capability of the conical insert in providing uniform test conditions over the entire test section and for a wide range of pressures has been experimentally verified. Figure 8 presents the pressure traces, acquired at different locations from the end-wall, for two exemplary (high- and low-pressure) postshock conditions: $p_{5}=5.1$ and 42.6 bar, respectively. The exact positions of the transducers are as follows: $x_{1}=851.1 \mathrm{~mm} ; x_{2}=650.9 \mathrm{~mm} ; x_{3}=316.5 \mathrm{~mm}$; $x_{4}=110.8 \mathrm{~mm}$; and $x_{5}=4.8 \mathrm{~mm}$.

As can be immediately deduced from Fig. 8, the pressure profiles remain nearly constant both in time and space for the experiments' typical duration of $4.5 \mathrm{~ms}$. The temporal and spatial uniformities of the thermodynamic parameters is a decisive factor for the accuracy of the measurements and, thus, represents a very important feature of the ITLR shock tube. 


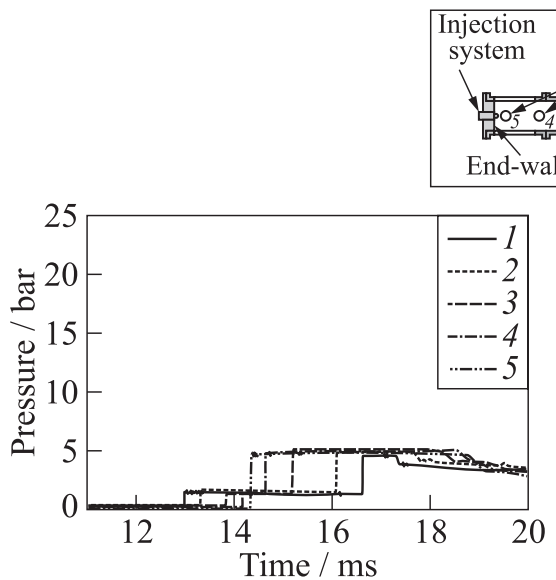

(a)

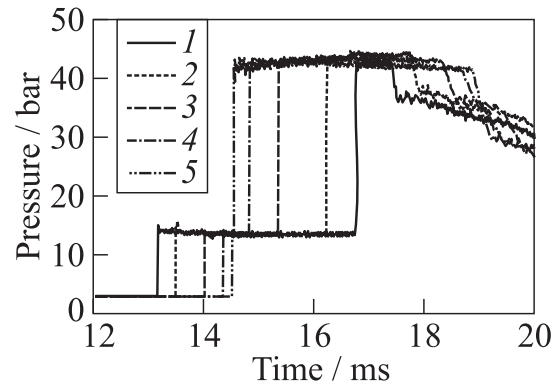

(b)

Figure 8 Temporal evolution of the pressure signal within the test section: $(a) p_{5}$ $=5.1$ bar; and $(b) p_{5}=42.6$ bar. Distance from end-wall: $1-851.1 \mathrm{~mm} ; 2-650.9$; $3-316.5 ; 4-110.8$; and $5-4.8 \mathrm{~mm}$

\subsection{Thermodynamic State of the Test Gas Behind the Reflected Shock Wave}

The thermodynamic properties of the test gas behind the reflected shock wave are the key parameters for most experiments conducted in the shock tube. In the jet disintegration and spray atomization experiments, e.g., the temperature $T_{5}$ strongly influences the evaporation process, the gas density $\rho_{5}$ affects the breakup process via shear and aerodynamic forces, and the pressure $p_{5}$ determines the nozzle exit velocity. Therefore, these quantities impact the experiments in many ways and their accurate determination is a prerequisite to a sound analysis and interpretation.

\subsubsection{Shock detection and velocity calculation}

As was shown in subsection 2.1, the test gas properties (state 5) are a function of the incident shock Mach number $\mathrm{Ma}_{s}$. The reflected shock Eqs. (1)-(3) can thus be applied to directly derive the postshock properties using the experimentally measured shock velocity (the shock Mach number). For this purpose, the pressure transducers, which are spaced along the shock-tube test section (see subsection 4.2 for more details), are used as they indicate the arrival of the incident shock at different axial positions. The procedures involved in the calculation of the test conditions were implemented in a Matlab ${ }^{\circledR}$ programme and are briefly discussed in the following paragraphs. 
After importing the pressure traces sampled at a rate of $1 \mathrm{MHz}$, a zero-phase digital filter with a window size of 8 is applied to reduce the noise level in the signals. After processing the pressure data in both forward and reverse directions, the resulting filtered data exhibited precisely zero-phase distortion, thus maintaining the original temporal accuracy. The actual shock detection was based on the calculation of pressure gradients, in which the incident and the reflected shock wave were clearly visible as pronounced spikes. The resolution of these spikes was increased hundredfold through a cubic spline interpolation, which modeled the shape of the pressure gradient traces and enhanced the identification of the peak gradient. Accordingly, the time of passage of the incident and reflected shock wave, respectively, was defined as the time instant at which the pressure gradient reached its local maximum, assuming that the transducers behaved similarly. The latter assumption seems valid as the "shape" of the shock wave in all pressure signals was very similar and a permutation of the sensors did not affect the results.

Typical pressure recordings and the corresponding pressure gradients are shown in Fig. 9. The dotted lines in Fig. $9 a$ indicate the determined passage of the incident shock wave. Note that the pressure trace of each transducer is shifted by 20 bar with respect to its successor. The five left spikes in Fig. $9 b$ mark the incident shock, the ones on the right hand side indicate the reflected shock.

The determined time differences $\Delta t_{i j}$ between the passage of the incident shock wave at the transducer positions and the known spacing $\Delta x_{i j}$ between the respective transducers was subsequently used to determine the local incident shock velocities $v_{i j}$. Having calculated the velocities, the calculation of the local incident shock Mach numbers $\mathrm{Ma}_{s, i}$ was straightforward by dividing by the speed of sound $a_{1}$.

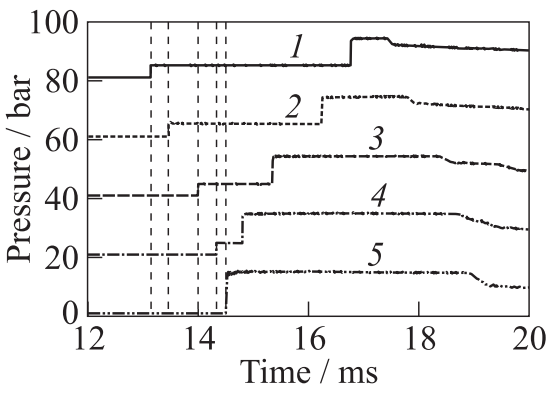

(a)

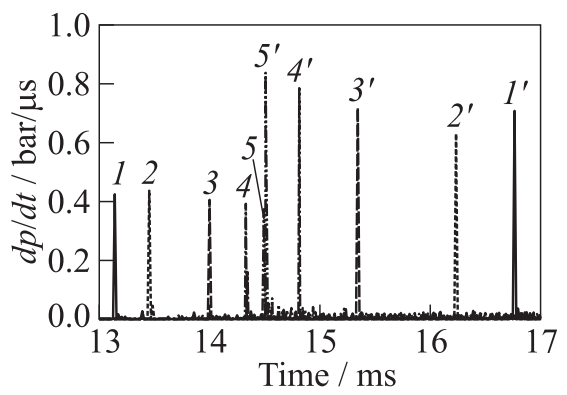

(b)

Figure 9 Typical pressure recordings and calculated times of shock passage (dotted lines) (a) and corresponding pressure gradients of the five pressure transducers mounted in the shock tube test section (b). Test conditions: $957.4 \mathrm{~K}, 15.0 \mathrm{bar}$, and $7.6 \mathrm{~kg} / \mathrm{m}^{3}$ 


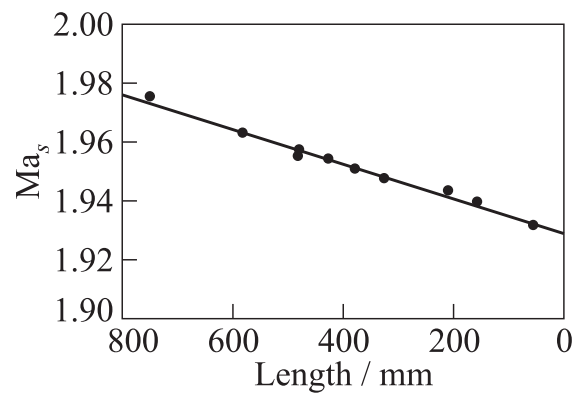

Figure 10 Typical measured axial Mach number profile and linear least-square fit. Length $=0 \mathrm{~mm}$ marks the position of the shock tube end-wall. Test conditions: $957.4 \mathrm{~K}, 15.0 \mathrm{bar}$, and $7.6 \mathrm{~kg} / \mathrm{m}^{3}$

Due to the nonideal effects described in subsection 2.2, the derived local shock Mach numbers $\mathrm{Ma}_{s, i}$ were not identical. The viscous effects led to boundary layer growth and slowed down the shock wave leading to monotonically decreasing shock Mach numbers. For the calculation of the postshock conditions, however, the Mach number at the end-wall must be known. The latter can be inferred by fitting a curve (usually, a straight line) to all local Mach numbers and extrapolating it to the end-wall. In Fig. 10, typical Mach number measurements are provided. The dots indicate all possible Mach numbers, which can be calculated using the five pressure transducers.

\subsubsection{Thermodynamic postshock conditions}

The properties straight after shock reflection $\left(p_{5.0}, T_{5.0}\right.$, and $\rho_{5.0}$, respectively) were calculated using the $1 \mathrm{D}$ normal shock Eqs. (1)-(3) with the calculated incident shock Mach number $\mathrm{Ma}_{s}$ at the end-wall and the initial fill conditions. Although these equations were derived for an inviscid, perfect gas assumption, they yield highly accurate results, as all losses are accounted for in the experimentally determined shock Mach number. Scaling of the pressure signals was necessary as the pressure transducers were shielded against heat transfer with a thin layer of silicone in order to avoid wrong pressure profiles. Applying this insulation method, the pressure readings were qualitatively correct. For quantitative pressure data, however, the original readings needed to be scaled using the $1 \mathrm{D}$ shock equation and the measured shock speed.

The pressure recording of the transducer closest to the end-wall was then used to determine pressure, temperature, and density profiles, applying the isentropic relation (Eq. (4)). This procedure is commonly accepted for shock tubes with no energy release and previous investigations showed the validity of this 
approach $[11,19,20]$. The driver insert used to compensate shock attenuation produced isentropic rarefaction waves solely; therefore, the isentropic assumption was not corrupted by its application. Furthermore, real-gas effects could be neglected, as the postshock conditions were constant and, thus, the change in compressibility could be entirely neglected [21].

The test time of the experiments was defined as the time of quasi-constant chamber conditions. Its end is marked by the pressure drop which can be identified in the pressure traces (see Fig. 9a), which stems from an expansion wave originating from the propagation of the reflected shock in a portion of the shock tube with a wider cross section. For higher driver-gas speeds of sound, the test time is shortened, as the main reflection of the rarefaction head arrives earlier in the test section, thus terminating the constant test conditions. During the period of quasi-constant conditions, the mean pressure, temperature, and density were calculated, resulting in the actual test conditions $p_{5}, T_{5}$, and $\rho_{5}$. For the experiments performed in this study, the driver gas composition was chosen in order to increase the test time without an excessive reduction in shock strength and consisted of an $85 \% \pm 3 \%$-helium $/ 15 \% \pm 3 \%$-argon mixture.

\subsubsection{Uncertainties in the reflected shock properties}

The previously described method of inferring the reflected shock properties in state 5 from the measured shock speeds and the initial fill conditions using normal shock equations is precise under the given assumptions of a planar shock, the exclusion of real-gas effects and similar behavior of all transducers involved. The uncertainty in these data, therefore, arises from the accuracy of the shock velocity measurements and the uncertainties in the initial fill conditions (1).

The average speed between any two points (here: pressure transducers) depends on two quantities: distance and time. These two quantities, however, are determined with certain accuracy; therefore, the derived speed also comprises some inaccuracy. The relative positions of the transducers are influenced by the position and the size tolerances of the main transducer ports, the size tolerances of the sensor adaptors, and the size and position tolerances of the small hole that houses the pressure transducer. The uncertainties in the measurements result from the resolution of the instruments used and an additional "handling" error, which in total yield $0.1 \mathrm{~mm}$. For a conservative estimate, an additional error of $0.1 \mathrm{~mm}$ was added to account for positioning and size tolerances in the adaptors, resulting in a total uncertainty of $0.2 \mathrm{~mm}$.

The uncertainties associated with the timing of the measurements can be divided into two categories: uncertainties in the triggering of the data acquisition cards and resolution uncertainties due to the limited sample rate of the recording system. The resolution uncertainties can be directly derived from the sample rate of the recording system and, thus, yield an error of $0.5 \mu \mathrm{s}$ which is half the time 
between two sample points. The four transducers closest to the end-wall are connected to one data acquisition card, the one furthest upstream to a separate one. Hence, the uncertainties arising from differences in the triggering instant concern only the latter sensor, when involved in the data reduction process. The triggering error is estimated to be $0.5 \mu \mathrm{s}$. Inspection of the pressure histories showed very similar behavior of all pressure transducers. Therefore, this error is reckoned to be markedly smaller than the other ones and an uncertainty of $0.25 \mu \mathrm{s}$ is used herein as a conservative value to account for possible differences in sensor behavior and, thus, in timing errors. The total uncertainties in time amount to $0.75 \mu \mathrm{s}$ for the four transducers closest to the end-wall, and to $1.25 \mu \mathrm{s}$, respectively, for the sensor furthest upstream.

For the determination of the reflected shock properties and the related uncertainties, which is the ultimate goal, the measurement of two additional quantities is required: initial test-gas temperature $T_{1}$ and pressure $p_{1}$. The uncertainty in the temperature $T_{1}$, which is also needed for the calculation of the shock Mach number $\mathrm{Ma}_{s}$, is $0.35 \mathrm{~K}$. The calibration certificate for the pressure sensor used to measure the filling pressure $p_{1}$ quotes a total error band of $\pm 0.009 \% \mathrm{FS}$; therefore, an uncertainty of 1 mbar is used for the calculations.

With the above estimates for the sources of error, the single uncertainties in all shock Mach numbers $\mathrm{Ma}_{s_{i j}}$ (derived between all transducer spacings) are determined using the Gaussian law of error propagation:

$$
\sigma_{R}=\sqrt{\sum_{i=1}^{n}\left(\frac{\partial R}{\partial x_{i}} \sigma_{x_{i}}\right)^{2}}
$$

Depending on the distance (and the timing errors) uncertainties of the order of $\sigma_{\mathrm{Ma}_{s_{i j}}}=1 \%$ arise. For the calculation of the errors in the extrapolated Mach number $\mathrm{Ma}_{s}$, which is used for the calculation of the actual test conditions, a Monte Carlo approach is used. For this purpose, a set of 10,000 normally distributed random data points is created $\left(\left(\mathrm{Ma}_{s_{i j}}\right)_{n}\right.$ with $\left.n=1 \ldots 10,000\right)$ for every Mach number $\mathrm{Ma}_{s_{i j}}$, with a mean which is the Mach number $\overline{\left(\mathrm{Ma}_{s_{i j}}\right)_{n}}$ $=\mathrm{Ma}_{s_{i j}}$ and a standard deviation $\left(\sigma_{\mathrm{Ma}_{s_{i j}}}\right)_{n}=\sigma_{\mathrm{Ma}_{s_{i j}}}$ which corresponds to the uncertainty in the specific Mach number. Subsequently, a straight line is fitted to all Mach number combinations and the wall Mach numbers $\left(\mathrm{Ma}_{s}\right)_{n}$ are derived. The final wall Mach number is the mean. The uncertainty is the standard deviation of all extrapolated Mach numbers.

Figure $11 a$ shows a sample result of the incident shock Mach number measurements with the extrapolated wall Mach number and error bars. The histogram of Monte-Carlo-simulated wall Mach numbers of the same experiment is given in Fig. 11. With the results of the Mach number measurements and the derived uncertainties, the uncertainties in the thermodynamic properties instantaneously behind the reflected shock wave $\sigma_{T_{5}}, \sigma_{p_{5}}$, and $\sigma_{\rho_{5}}$ can be calculated again, in- 


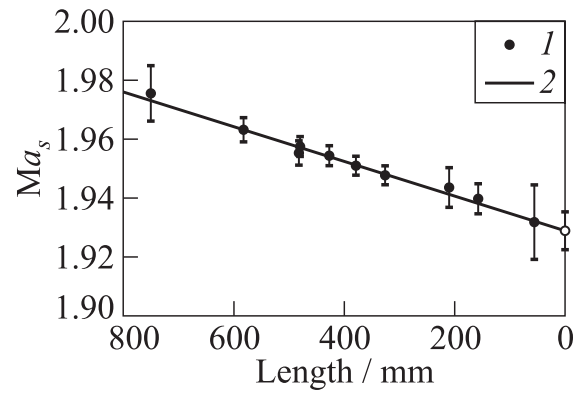

(a)

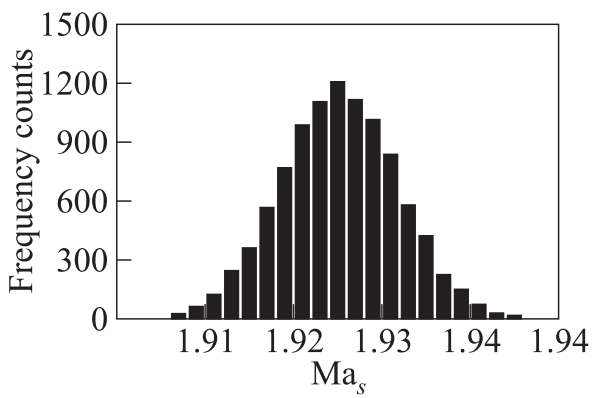

(b)

Figure 11 Sample shock Mach number measurements: (a) measured shock Mach numbers with calculated error bars and linearly extrapolated wall Mach number; and (b) histogram of the simulated wall Mach numbers using a Monte Carlo approach $\left(\mathrm{Ma}_{s}=1.925 ; \sigma_{\mathrm{Ma}_{s}}=0.0065\right)$. Test conditions: $957.4 \mathrm{~K}, 42.1 \mathrm{bar}$, and $21.1 \mathrm{~kg} / \mathrm{m}^{3}$

voking the law of error propagation. For all properties, the uncertainties amount to less than $2 \%$.

\section{OPTICAL SETUP AND IMAGE ANALYSIS}

\subsection{Schlieren Setup}

The actual geometrical arrangement of the optical setup used for the Schlieren imaging experiments is shown schematically in Fig. 12.

A short-pulsed high-power light emitting diode (LUXEON Rebel LXMLPWC1-0100) was used as the light source (luminous flux of $180 \mathrm{~lm}$ at the nominal drive current $(700 \mathrm{~mA})$ and voltage $(3.4 \mathrm{~V}))$. The emitted light was collimated by an achromatic field lens $(f=160 \mathrm{~mm}, D=80 \mathrm{~mm})$ which produced a beam with a diameter slightly larger than the diagonal of the test chamber windows. This light beam was passed perpendicularly to the injection axis through the test chamber of the shock tube where it back-illuminated the spray. It was subsequently collected and focused by a slightly tilted (approximately $9^{\circ}$ ) parabolic field mirror $(f=900 \mathrm{~mm}, D=150 \mathrm{~mm}$ ), located $1800 \mathrm{~mm}$ downstream from the middle of the test chamber, i. e., at the injection axis. A Schneider-Kreuznach Super-Symmar HM 5.6/150 objective lens served as the focusing lens to form the final Schlieren image. Its aperture was placed in the focal point of the field mirror, hence acting as a Schlieren cutoff. Schlieren images were recorded using a Photron Fastcam SA1 high-speed camera.

At full resolution $(1024 \times 1024)$, the high-speed camera was able to record images at a frame rate of $5.400 \mathrm{fps}$, its minimum shutter time was $1 \mu \mathrm{s}$. In order to increase the actual acquisition frame rate, a $512 \times 160$ pixels section 


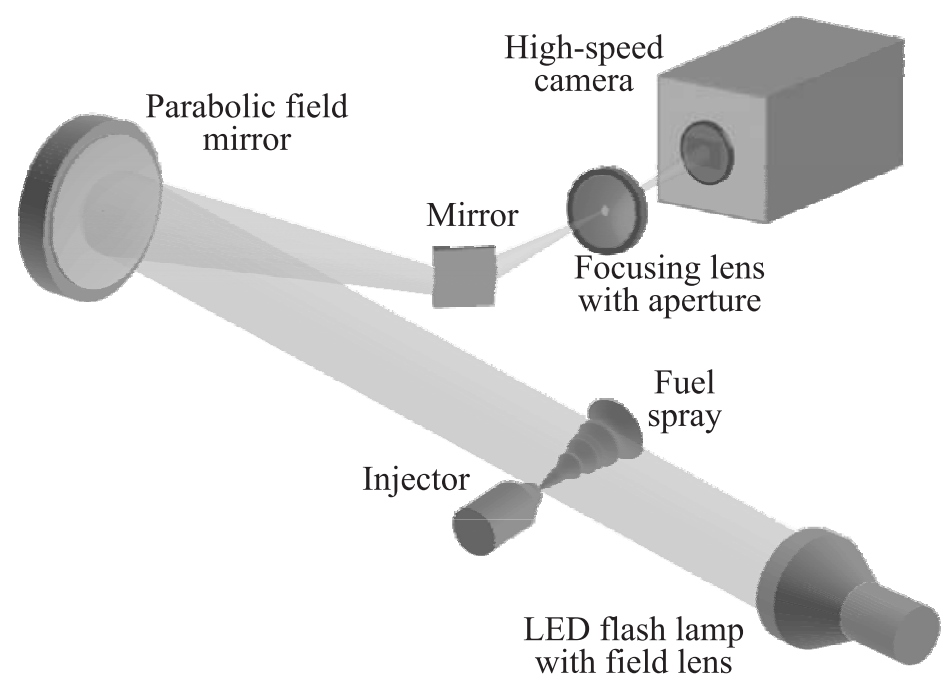

Figure 12 Schematic layout of the Schlieren setup

of the camera chip was used, yielding frame rates of $62.500 \mathrm{fps}$. The optical setup resulted in a magnification factor $M$ of about $1: 6$, which corresponded to an effective optical resolution of about $120 \mu \mathrm{m} /$ pixel. The pixels of the camera have an edge length of $20 \mu \mathrm{m}$ and the LED lamp produced light pulses with a width of $200 \mathrm{~ns}$ (FWHM). These parameters yielded a maximum theoretical velocity without motion blur of about $600 \mathrm{~m} / \mathrm{s}$, i. e., at this velocity an image was shifted by one pixel. In the present study, the velocity of the spray was always $\leq 200 \mathrm{~m} / \mathrm{s}(1 / 3$ of the maximum velocity allowed); therefore, sharp images of jets could be acquired without motion blur. The initial shock wave velocity $u_{s}$ was of the order of $600 \mathrm{~m} / \mathrm{s}$; hence, a small amount of motion blur might be present in images in which shocks were displayed. Synchronization of camera and light source was achieved by slaving the light source to the internal clock of the high-speed camera.

Finally, the luminescence distribution in the final recorded images was due to two effects: $(i)$ the Schlieren effect caused by gradients in temperature and fuel concentration (i.e., gradients in the refractive index); and (ii) extinction. The latter occured mainly in the dense spray region where light, which was primarily scattered from the liquid in the spray, was removed from the optical path.

\subsection{Planar Mie-Scattering Setup}

The optical setup used for the planar Mie-scattering experiments is shown in Fig. 13. As the light source, a Nd:YAG continuous wave (cw) laser (Spectra- 


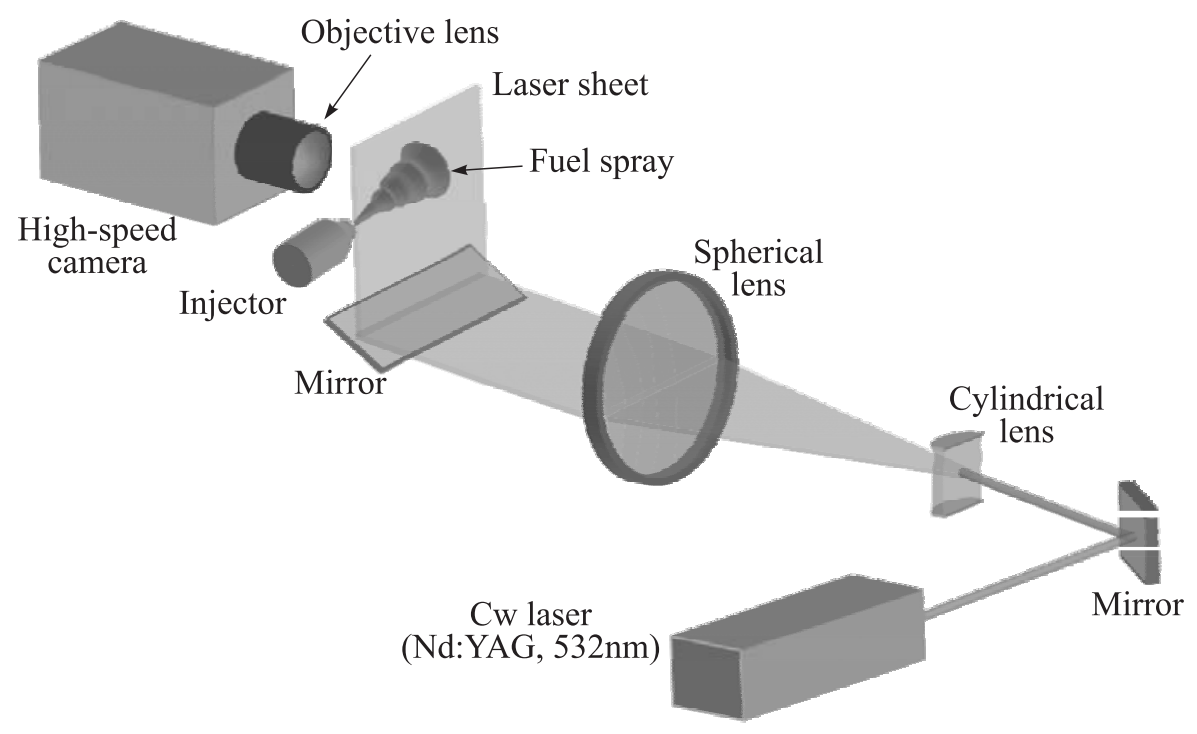

Figure 13 Schematic layout of the Mie imaging setup

Physics Millennia Pro 5s) with a wavelength of $532 \mathrm{~nm}$ was used. The laser was operated at a nominal output power of $2 \mathrm{~W}$ and its beam diameter (measured at the exit port) was $2.3 \mathrm{~mm}( \pm 10 \%)$. The emitted round laser beam was formed into a laser sheet by passing it through a set of lenses. First, the beam was expanded in the horizontal direction by a convex cylindrical lens with a focal length of $f=10 \mathrm{~mm}$. This widened up laser band was then collimated and focused by a spherical lens ( $f=500 \mathrm{~mm}, D=60 \mathrm{~mm}$ ), located $500 \mathrm{~mm}$ (one focal length of the spherical collimation lens) downstream from the focal point of the cylindrical lens, thus forming a thin laser sheet. The lens was mounted in a blackened lens mount, which also served as an aperture to transmit only the middle part $(\approx 50 \%$ ) of the laser sheet in order to increase its spatial uniformity. The resulting horizontally oriented sheet was then deflected upwards by $90^{\circ}$ using a plane mirror and coupled into the test chamber through the floor window. Its location coincided with the vertical midplane of the test chamber (the injection axis, respectively) in order to illuminate only the vertical symmetry plane of the fuel jet.

The scattered light images were recorded with the high-speed camera, operated at similar settings as for the Schlieren experiments, yielding images with a resolution of $512 \times 160$ pixels, frame rates of $62,500 \mathrm{fps}$ and a magnification factor $M$ of about $1: 6$. However, as the laser was not pulsed, the shutter time was limited to the minimum shutter time of the camera $(1 \mu \mathrm{s})$; therefore, slight motion blur might occasionally occur (maximum shift by one pixel). The ob- 
jective lens used for the Mie-scattering experiments was a standard macrolens (Nikon AF MICRO NIKKOR D $60 \mathrm{~mm} / 1: 2.8$ ).

\subsection{Processing of High-Speed Images}

\subsubsection{Macroscopic Schlieren images}

Image Cropping. The first step of image processing was to determine which image regions were of interest with respect to further analysis (i. e., regions which contained the fuel jet and the surrounding gas) and which regions showed perturbing objects such as the injector head or parts of the test chamber window frames. It was found that a fast and reliable way was to calculate a mean grey value within a reference region (the first four pixel columns) in the first image where only the end flange (i.e., the left part of the window) was visible and to use this as a reference grey value $I_{\text {inj }}$. Thereafter, the minimum grey value $I_{j \text {,min }}$ in each pixel column $j$ was calculated and compared with the reference value from which the location of the injector head could then be deduced. All images were then cropped at this point and at the right-hand window frame, so that only the region of interest remained.

Spatial Intensity Correction. The illumination system used was characterized by a very good spatial uniformity and temporal constancy of the light source. However, small fluctuations both in space and in time could not be completely eliminated. The spatial intensity deviations (typically $<2 \%$ from the mean) could be attributed to the spatial radiation pattern of the LED according to Lambert's cosine law. To account for this, all images of a test-sequence were spatially normalized by a prefiltered reference image of the same image section (usually the first image of the sequence). Thus, the normalized local intensity $I_{\text {norm }}$ for every pixel $(i, j)$ was given by

$$
I_{\text {norm }}(i, j)=I_{0}(i, j)\left(\frac{I_{\max }}{I(i, j)}\right)_{\text {ref }}
$$

where $I_{0}(i, j)$ is the initial raw image intensity of every pixel and $\left(I_{\max } / I(i, j)\right)_{\text {ref }}$ is the reciprocal of the normalized background (reference) intensity. Besides the intensity correction, any present image fouling was also effectively removed by this procedure.

Adaption of Absolute Intensity. After the normalization procedure, the intensity range of the images was adapted, so that the full span from 0 to 16 bit was used. This ensured the direct comparability between all experiments as the background intensities of all images of an experimental series were set to a similar value and the images of all experiments covered the same dynamic range.

Subpixel Interpolation. Finally, subpixel interpolation was used to increase the spatial resolution of the images and to ensure equal resolution of all images, 
as they were recorded during several experimental campaigns leading to slightly different magnification factors $M$ depending on the specific campaign. Besides the improved visual appearance of the images, subpixel interpolation led to refined spray contours, less error-prone spray identification, and thus, to more accurate results. The improved accuracy was particularly relevant for the region closer to the nozzle, where a shift of the spray contour by a single pixel might cause high deviations from the "real" value.

Image Binarization and Identification of Jet/Spray Contours. The definition of the spray contour required the accurate separation of the spray from the background. Unfortunately, the contour of an atomizing and evaporating spray was not clearly confined, as it consisted of a mixture of liquid fuel, fuel vapour, entrained ambient test gas, and, depending on the test conditions, supercritical fluid. The spray contour marked the smooth transition between the spray and the surrounding gas and, on this account, its definition was influenced to some extent by the selected greyscale/intensity value at which the spray images were thresholded. Thus, the determination of a representative threshold value was of importance for the accuracy and the interpretation of the results.

The distinction between spray and background was becoming more tedious with worsening the signal-to-noise ratio (i. e., the spray-to-background intensity ratio). Thus, the dilute spray regions with rather high grey values, which were comparable to those of the actual background, exhibited a lower contrast and were, therefore, harder to separate from the background than the dark, dense spray regions. Furthermore, very noisy images with strong intensity fluctuations in the background deteriorated the spray contour recognition as the intensities occurring in the spray and in the background could be of the same order.

The binarization procedure followed a two-stage approach and took into account the background noise level of the specific image series. At the first step, all images were thresholded at a distinct level ( $75 \%-95 \%$ of the background mean), which was chosen so as to ensure that the full spray was captured and could thus be broadly isolated. From these intermediate binary images, the horizontal and vertical extensions of each spray were calculated and the images were cropped at these positions. Thus, all dispensable background pixels were omitted and the ratio of spray-to-background pixels was similar for all images.

The actual binarization was performed after enhancing the contrast of the cropped images by histogram equalization. The purpose for this was to increase the distinguishability in the regions where spray vapour and surrounding gas showed very similar intensities and could, otherwise, not be separated from each other. The thresholds were automatically calculated using Otsu's method [22]. The latter calculates an ideal threshold by minimizing the intraclass variance, i. e., the variance of the intensity values within each class contained in the resulting binary image is minimal, whereas the spread between those two classes is maximized. Additionally this method was applied for two regions in every image, 


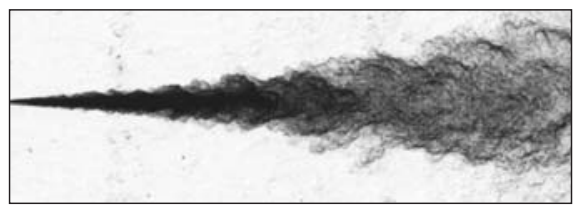

(a)

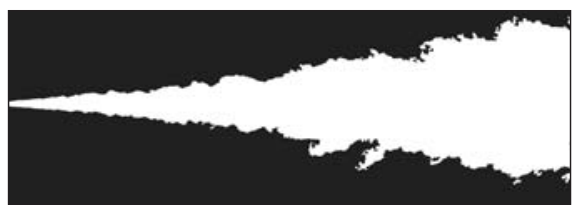

(c)

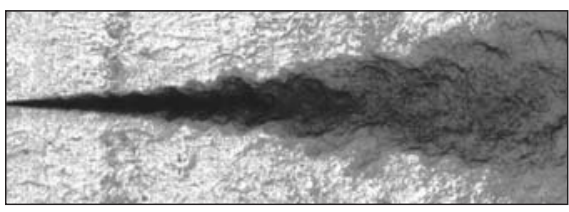

(b)

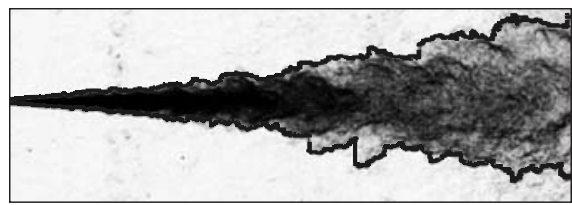

(d)

Figure 14 Procedure of the image binarization showing intermediate images and final spray contour: $(a)$ original image; $(b)$ image after histogramequalization and recombination with original image; $(c)$ binary image; and $(d)$ detected spray contour overlayed with the original image

the far field and the near field (with respect to the nozzle), in order to account for local differences in the image histograms. The described procedure was capable of successfully and robustly separating the spray (liquid and nonliquid parts) from the background and reliably coped with challenging issues such as strong background noise, low contrast, or interfering particles (e.g., diaphragm splinters). The contour of the spray was then defined as the trace of the outermost pixels belonging to the fuel jets. The different stages of the contour detection procedure are shown in Fig. 14.

\subsubsection{Planar Mie-scattering images}

The planar Mie-scattering images were treated in a very similar way as the Schlieren images. As this type of images represented a sort of "negative" of the Schlieren images (dark background, bright spray), they first had to be inverted for obtaining complementary images. Due to the measurement technique, where light was scattered at liquid particles, the images experienced virtually no background noise, thus filtering of the images was not necessary. As for the Schlieren images, the jets were separated from the background by a thresholding method.

The procedure previously described for the Schlieren images was not feasible for the Mie-scattered images, as the threshold calculated with Otsu's method usually strongly underestimates both the width (i.e., the diameter) and the penetration length of the liquid phase in these images. Therefore, the threshold was chosen by visual inspection of many images acquired for several conditions, 
especially for those where large differences in the results were to be expected. Of course, a certain influence of the threshold level on the results could be observed.

For the selection of a "suitable" threshold, the following criteria have been adopted. Rather small threshold levels were not considered as they obviously neglected larger parts of the liquid spray region and were, therefore, unrealistic. Very large threshold values, in turn, might lead to unwanted effects in the binary images: contamination of the observation windows, e. g., is a source of stray light, and might lead to a reduction in the signal-to-noise ratio. This spurious light could be suppressed by choosing a threshold value, tuned on the intensity of the spray signal, which was generally much higher. In this study, the threshold level for the Mie-scattered images was chosen to be $95 \%$, giving meaningful results with acceptable uncertainties for all conditions.

\subsubsection{Definition and calculation of jet parameters}

In the evaluation of spray characteristics, the following macroscopic parameters were considered: (local) jet spreading angle, spray tip penetration length/

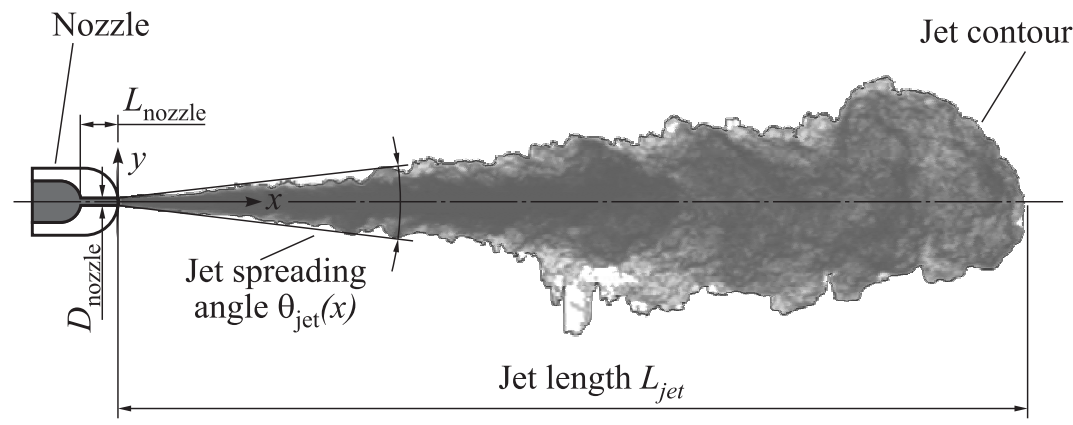

(a)

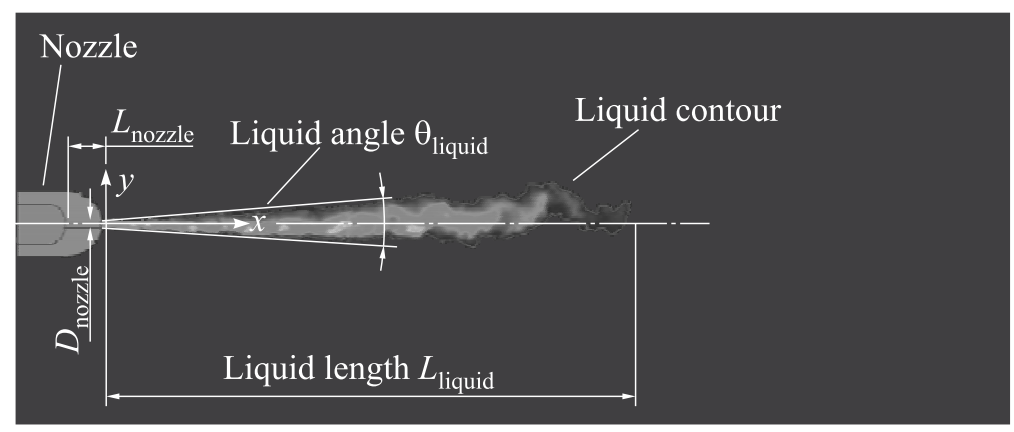

(b)

Figure 15 Definition of jet parameters 
velocity (in the start-up phase) and liquid length. Their geometrical definition is illustrated in Fig. 15.

Jet Spreading Angle. In the literature, there is no general consensus on the definition of the jet spreading angle $\theta_{\text {jet }}$. A common approach is to calculate the spreading angle from the spray width at a defined axial position (see, for example, $[23,24])$ or to calculate the equivalent angle of an isoarea triangle [25]. More global definitions, which take a considerable part of the spray contour into account, can also be found in [26]. This variety of definitions considerably complicates the comparison between results.

In the present work, the axial distribution of the local spreading angle $\theta_{\text {jet }}$ $=\theta_{\text {jet }}(x)$ was considered, as it provides a better physical insight on the spatiotemporal evolution of the spray and its interaction with the surrounding gas. The spreading angle, in fact, is directly related to the rate of gas entrainment by the jet and is influenced by the forces acting on the jet at the nozzle exit. Depending on the chamber and injection conditions, the local spreading angle may vary along the axis.

The jet/spray contour actually consists of an upper and a lower branch $y_{i}(x)$ which are subsequently used to calculate the upper and lower half of the local spreading angles $\theta_{i}(x)$ of the fuel jet. Starting at the nozzle exit and ending at a defined axial distance $x$, the (local) spreading angle $\theta_{i}(x)$ can be calculated employing the equation:

$$
\tan \left[\theta_{i}(x)\right]=\frac{y_{i}(x)-y_{i}(1)}{\Delta x} \quad(i=\text { upper, lower }) .
$$

The calculation of the full spreading angle $\theta_{\text {jet }}(x)$ is straightforward by combining the "half" angles of the upper and lower contour to the full angle:

$$
\theta_{\text {jet }}(x)=\theta_{\text {upper }}(x)-\theta_{\text {lower }}(x) .
$$

Applying Eq. (5) to all acquired images, temporally and spatially resolved information on the spray angle is gained. Besides, a "liquid" angle $\theta_{\text {liquid }}$ can be derived in the same manner from the Mie scattered images to describe the distribution of the liquid phase.

Jet/Spray Length. The jet/spray tip penetration $L_{\text {jet }}$ (or axial growth rate) during the start-up process of the injection was calculated from the axial distance of the Schlieren spray contour from the nozzle exit. Specifically, the maximum axial location $x_{\max }$ of the contour was calculated and defined as the spray tip length $L_{\text {jet }}(t)$. Using the spray lengths of all images and the chosen frame rate of the camera, the (transient) velocity of the spray tip $v_{\text {jet }}$ could be derived.

Jet/Spray Liquid Length. The liquid core length $L_{\text {liquid }}$ of a jet is a measure for the axial penetration of the jet/spray core into the environment. The "liquid core" can be either specified as the intact core (connected liquid) or as the dense 
core (high liquid/gas density ratio). Previous work (see, e.g., [27]) showed that after the initial phase, the liquid core remained at a quasi-steady length where only small fluctuations caused by turbulence were observed. The quasi-steady liquid core length was then chosen as the representative value for each condition.

\section{RESULTS OF THE JET DISINTEGRATION EXPERIMENTS}

The objective of this section is to demonstrate the feasibility of near-critical jet disintegration studies in the ITLR shock tube. For this purpose, two different disintegration regimes (i. e., the transcritical and supercritical regimes) were studied in details and the results were compared to the literature data. The ambient conditions were always supercritical in temperature $\left(T_{\mathrm{chm}}=957 \mathrm{~K} \pm 6 \mathrm{~K}\right)$, while the ambient pressure was varied from subcritical (10 bar, $p_{r}=p_{\text {chm }} / p_{c}=0.33$ ) to supercritical values $\left(42.5 \mathrm{bar}, p_{r}=1.40\right)$. For reference, the critical temperature and pressure of $n$-hexane are $507.9 \mathrm{~K}$ and 30.35 bar, respectively. The tests were performed using a "cavitation-free" nozzle with an inner diameter of $D=236 \mu \mathrm{m}$. The disintegration process was visualized by means of macroscopic high-speed Schlieren and Mie-imaging. The data postprocessing always included a comparative analysis of both sets of images in order to analyze the jet structure and identified liquid areas within the transcritical/supercritical jet.

Due to its relevance to the interpretation of the shock tube data, it is useful to make a brief discussion on the scattering of light from matter. Light can be scattered by molecules or density fluctuations in the gas phase (Rayleigh scattering) or by particles, whose dimensions are comparable with the wavelength of light (Mie scattering). The former is observed in supercritical regions, and the latter is dominant in regions where scatterers (or, in general, an interface between two distinct phases) are present. Due to the high intensity difference between Mie scattering from droplets and Rayleigh scattering, the latter requires the use of an intensified charge-coupled device (CCD) camera and/or photomultipliers to be detected (see, e.g., [28]). Due to the poor light sensitivity of high-speed cameras, the only scattered light which can be detected in the present application is due to Mie scattering, which becomes, therefore, an indicator of subcritical liquid areas within the jet.

\subsection{Transcritical Regime}

In the transcritical regime, the occurrence of thermodynamic and transport anomalies, associated with the local transition to a supercritical state, may have a strong impact on the dynamics of (liquid) jet disintegration and on the mixing process. Early investigators (see, e.g., [4, 29, 30] for reference) observed 
finger-like entities at the interface of a liquid jet, embedded in a supercritical environment. These entities did not break up into droplets as in standard atomization and formed a mixing layer in which local transition to a supercritical state and/or local density nonuniformities occurred. These observations led to the conclusion that the disintegration process resembled the injection of a gaseous turbulent jet into a gaseous environment. To support this hypothesis, Chehroudi et al. [31] analyzed the dependence of the jet spreading angle $\theta_{\text {jet }}$ upon the density ratio $\rho_{\mathrm{g}} / \rho_{\mathrm{l}}$ and compared it with the growth rate of liquid sprays, variable-density gaseous jets and mixing layers. The analysis showed that, for the range of density ratios in which gas-like appearance was observed, the experimental data agreed well with the theoretical equation describing the lateral spreading of compressible turbulent shear layers. Thus, it was concluded that at ambient condition exceeding the critical point of the injectant, the sprays had not only the same appearance as gas jets, but the spreading rates of the two flows were quantitatively the same.

More recently, several authors (see, e.g., [2, 3, 32, 33]) have questioned the similarity in mixing behavior between a transcritical spray and a turbulent gaseous jet and/or mixing layer. The main objections can be summarized as follows. First, the increased relative importance of thermodiffusional effects reduces mass diffusion, induces density gradients in the shear layer, and inhibits mixing. Due to these factors, the spray has a much longer unmixed core length compared to the widely recognized and accepted turbulent gaseous jet theory by Abramovich [34]. Second, the analysis of shadowgraph/Schlieren images can be misleading due to the fact that both techniques measure only density gradients. Therefore, they are unable to distinguish between subcritical (i. e., liquid) and supercritical areas within the spray. Third, the rapid increase in specific heat near the critical point drastically reduces the heat transfer process from the hot surroundings towards the inner core of the spray. This implies that a "cold" (subcritical) liquid core may persist undisturbed during the spray disintegration process.

To investigate the (potential) transition to supercritical fluid and its impact on the jet structure, three different fuel injection temperatures were considered: $295 \mathrm{~K}\left(T_{r}=0.58\right), 449 \mathrm{~K}\left(T_{r}=0.88\right)$, and $522 \mathrm{~K}\left(T_{r}=1.03\right)$, respectively. Figure 16 shows the influence of chamber pressure on the evolution of liquid sprays initially at ambient temperature $T_{\mathrm{inj}}=295 \mathrm{~K}\left(T_{r}=0.58\right)$. At low reduced temperatures, subcritical spray disintegration resembles closely "standard" atomization, as shown in Fig. $16 a$ (left). The term "standard" atomization refers to the fact that the spreading angle increases with an increase in chamber density. This represents an established trend for the atomization regime, in agreement with the experimental observations, e. g., in $[24,35]$. This is due to the fact that as the ambient pressure increases, the velocity fluctuations in the radial direction become more vigorous. Large-scale instability waves develop in the near-injector region, which then grow up and roll into a succession of ring vortices as the 
injected fluid moves downstream. The resultant vortical flow motions facilitate the entrainment of the ambient gas into the cold spray fluid, thus resulting in an enhancement of the spray lateral spreading. The effect of density stratification on the evolution of mixing layers was studied both experimentally and numerically in $[3,36]$. All authors showed that higher density stratification increasingly inhibited instability-wave growth and vortex paring, while the flow topography was considerably simplified. Figure $16 a$ (right) presents the growth rate of the liquid core which is roughly half the size of the total spray spreading angle. Figure $16 b$ (left) depicts the liquid core length which is a decreasing function of chamber density. The reduction in liquid penetration is due to the larger lateral spreading of the spray and the enhanced vaporization, thanks to the facilitated entrainment of hot surrounding gas. Note that at $p_{r} \geq 0.9, L_{\text {liquid }}$ stabilizes around a constant value despite the increased quote of entrained hot gas within the sparay. Summarizing, in the near-nozzle region, the spray structure can be divided in an outer gaseous shear layer and an internal liquid core, whose axial extension reduces drastically with pressure. In agreement with the theoretical works $[3,36]$, the present authors' experimental results corroborate the statement that density stratification inhibits atomization. The far-nozzle region possesses, instead, all features of a turbulent gaseous jet.

The spray tip length during the first phase of fuel injection is given in Fig. $16 b$ (right). During the first 300-400 $\mu \mathrm{s}$, the penetration and, thus, the velocity of the spray tip is very similar for all pressures investigated. At this point, the spray tip gets noticeably decelerated at $p_{\mathrm{chm}} \geq 20$ bar, while it remains a fairly constant velocity at lower pressures. After about $800 \mu \mathrm{s}$, an observable difference is also found for all pressures. Spray tip velocities can, thus, be assumed to be constant at the initial stage of spray propagation (inertia-dominated regime). After a certain time $t_{0}$, which is referred in [35] to as the break-up time of the spray, the spray is remarkably slowed down. This deceleration is stronger for higher chamber pressure (density) and occurs earlier as the resistance of the gas is higher. The spray tip penetration, as well as the time of the transition between the linear behavior and the attenuated growth, decreases with increasing chamber pressures. The latter considerations on the spray tip penetration hold independently of the specific value of the initial fuel temperature.

As the reduced temperature increases (i. e., $T_{r}=0.88$ ), both the penetration of the liquid core and liquid spray angle are only slightly reduced compared to the "cold" spray values, as can be deduced from Fig. 16c (right). Both quantities remain a decreasing function of chamber pressure, albeit the pressure dependence becomes less pronounced as the ambient pressure approaches and/or exceeds the critical pressure of the fuel. Concerning the total lateral spreading of the spray, the disintegration process can be divided in two distinct zones. In the downstream region $(x / D \geq 22=5.2 \mathrm{~mm})$, the spray angles increase with a nearly linear slope. As a general trend, higher pressures and densities lead to larger spray angles at all axial positions. The position at which the curves 

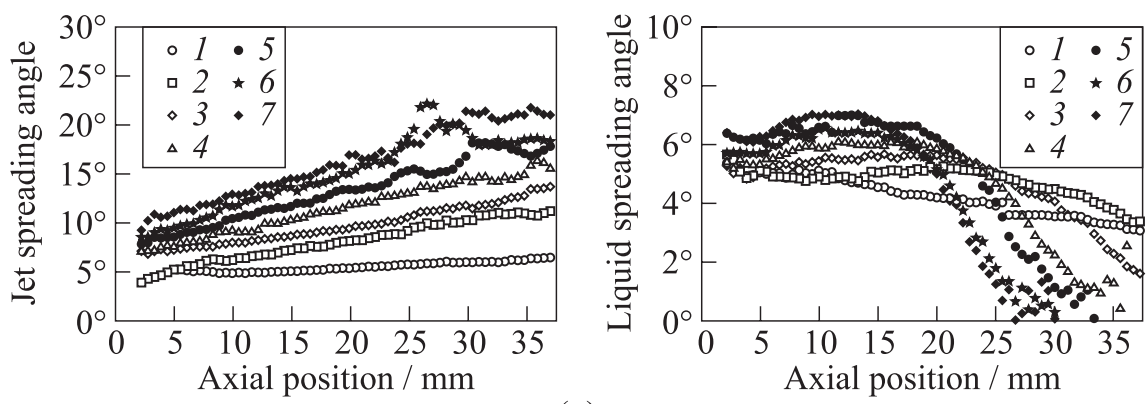

(a)
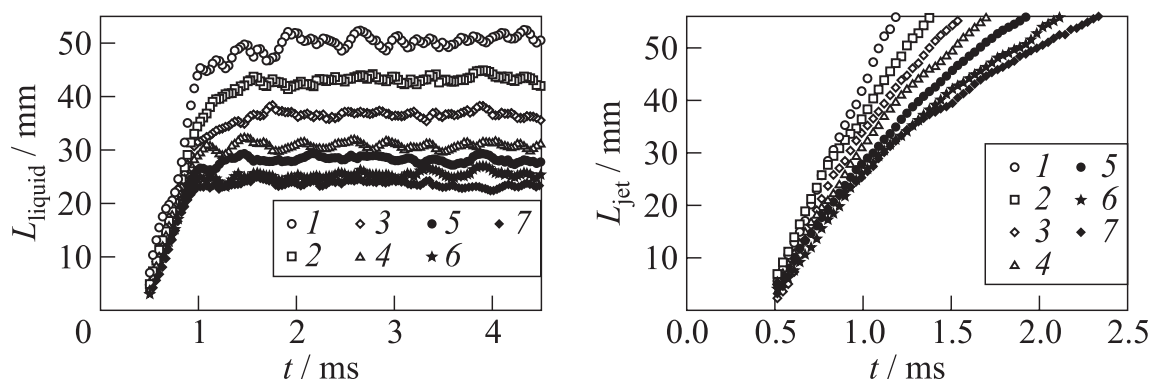

(b)
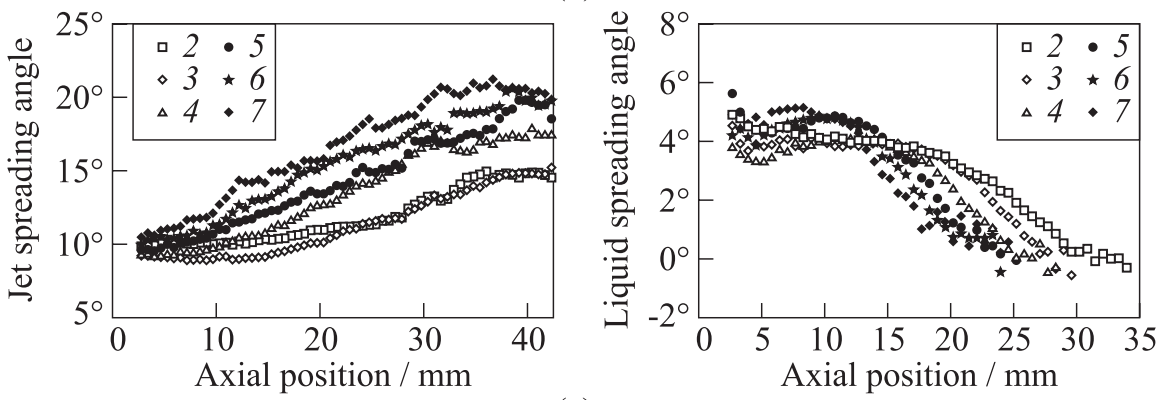

(c)

Figure 16 Influence of chamber pressure on the spreading angle $\theta_{\text {jet }}$, liquid spreading angle $\theta_{\text {liquid }}$, liquid core length $L_{\text {liquid }}$, and spray tip length $L_{\text {jet }}$ : $(a)$ and $(b) T_{r}$ $=0.58 ;(c) T_{r}=0.88 ; p_{r}$ is equal to 0.16 (1), 0.33 (2), 0.49 (3), 0.66 (4), 0.91 (5), $1.15(6)$, and $1.40(7)$; fuel $-n$-hexane: $(a)$ time averaged spreading angle $\theta_{\text {jet }}$ (left) and liquid spreading angle $\theta_{\text {liquid }}$ (right) vs. axial distance, $T_{f}=295 \mathrm{~K}$; $(b)$ liquid tip length $L_{\text {liquid }}$ (left) and spray tip length $L_{\text {jet }}$ (right) vs. time, $T_{f}=295 \mathrm{~K}$; and (c) time averaged spreading angle $\theta_{\text {jet }}$ (left) and liquid spreading angle $\theta_{\text {liquid }}$ (right) vs. axial distance, $T_{f}=449 \mathrm{~K}$ 

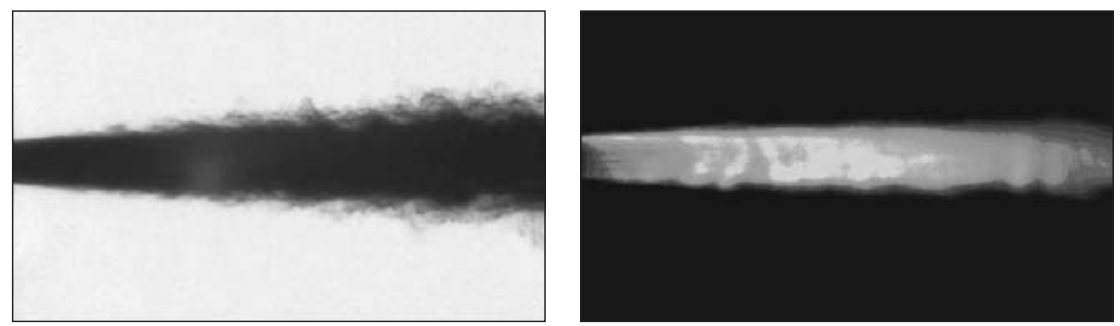

Figure 17 Structure of a supercritical jet; test conditions: $T_{f}=522 \mathrm{~K}, T_{\text {chm }}$ $=957 \mathrm{~K}, p_{\mathrm{chm}}=42.5 \mathrm{bar}, T_{r}=1.03$, and $p_{r}=1.40$; fuel: $n$-hexane

start to diverge coincides approximately with the onset of the breakup region at $t=t_{0}$ (breakup time, following the terminology of [35]). At $t>t_{0}$, the spray is slowed down and the influence of higher chamber pressure becomes more relevant through the enhanced gas entrainment. This region, further downstream of the nozzle tip, is dominated by turbulent shear-layer mixing effects.

In the near-nozzle region (i. e., $20<x / D<0$ ), the spray angle is only weakly dependent upon the ambient pressure and all curves approach a value around $10^{\circ}$. At supercritical ambient pressures (i.e., $p_{\text {chm }} \geq 30.2$ bar or $p_{r} \geq 0.96$ ), the growth rate curves approach the theoretical/experimental curves determined for fully turbulent, compressible shear/mixing layers [37, 38]. This virtually means that $\tan \left(\theta_{\text {jet }}\right) \approx 0.2$ already at the values of the ambient-gas-to-fuel density ratio of the order of 0.02 (i.e., $\rho_{\mathrm{g}} / \rho_{l} \approx 0.02-0.03$ ). A similar behavior was also found experimentally in [31] and marked as the onset for the transition from secondwind induced breakup to supercritical disintegration mode. However, a visual inspection of Fig. 17 reveals important information on the spray structure at near-critical conditions, which is composed of an inner liquid core surrounded by a shear transitional mixing layer. The persistence of the inner liquid core, even as the injection conditions approach the critical point, may be explained in light of the reduced heat transfer rate between the surroundings and the center of the spray due to thermodynamic anomalies. This experimental evidence confirms the theoretical/computational analysis of the authors of $[2,3,32,33,39]$, who raised doubts on the similarity in mixing behavior between a transcritical spray and a turbulent gaseous jet and/or mixing layer on the basis of thermodynamic considerations.

\subsection{Supercritical Regime}

The objective of this section is to characterize the expansion of a supercritical fuel, whose injection temperature is slightly above the critical value and with chamber pressures varying from supercritical to subcritical values. The section 


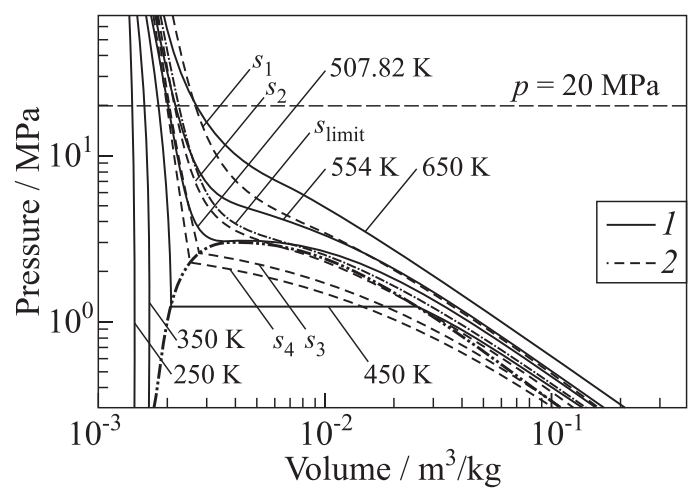

Figure $18 p, v$-diagram for $n$-hexane: $1-T=$ const; and $2-s=$ const

begins with a phenomenological description of the supercritical disintegration process, then the effects of ambient pressure and fuel injection temperature on the spray visual characteristics are presented. Finally, the available (literature) quantitative data on expansion angles and Mach disk properties are discussed.

The injection of a supercritical fluid in a subcritical and/or supercritical environment usually resembles that of a gaseous jet. Still, under particular circumstances, the fluid may enter the two-phase region during its expansion through the nozzle and nonequilibrium condensation may take place. This situation is depicted schematically in Fig. 18 for two hypothetical injection events at $p_{\text {inj }}=200$ bar and two supercritical temperatures $T_{\mathrm{inj}}=650$ and $554 \mathrm{~K}$, respectively. Assuming an isentropic expansion along the curve $s_{1}$ through the nozzle, it is clear that for an injection temperature $T_{\mathrm{inj}}=650 \mathrm{~K}$, the spray flow never enters the two-phase region and the injection/mixing process is similar to a gaseous mixing layer, even for very low chamber pressures. For injection temperatures $T_{\mathrm{inj}}>T_{c}$ and depending upon chamber conditions, the isentropic expansions $s_{2}-s_{4}$ may penetrate into the two-phase fluid region as long as the isentropic curves stay below $s_{\text {limit }}$. This is, for example, the case for the injection temperature $T_{\mathrm{inj}}=554 \mathrm{~K}$, which just intercepts the two-phase boundaries and corresponds to the image in Fig. $19 b$.

The inception of a liquid phase not only affects drastically both the mixing process and the ignition of the fuel, but has also a strong impact on the visual characteristics of the spray and results in a much longer fuel core and in an increased lateral spreading. Figure 20 (courtesy of [40]) provided the first experimental evidence of this phenomenon. It shows two shadowgraph images of a supercritical methane/ethylene spray at different injection temperatures with and without fuel condensation. At higher injection temperature $\left(T_{r}=T_{\mathrm{inj}} / T_{c}=1.23\right)$, the spray appearance is transparent, with a clear shock structure visible in the shadowgraph image. The dome-shaped spray boundary 

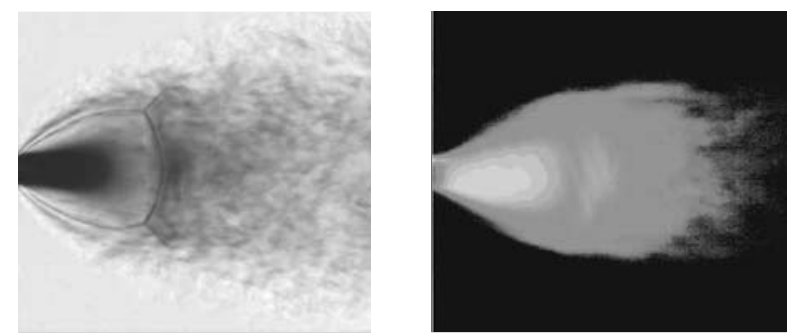

(a)
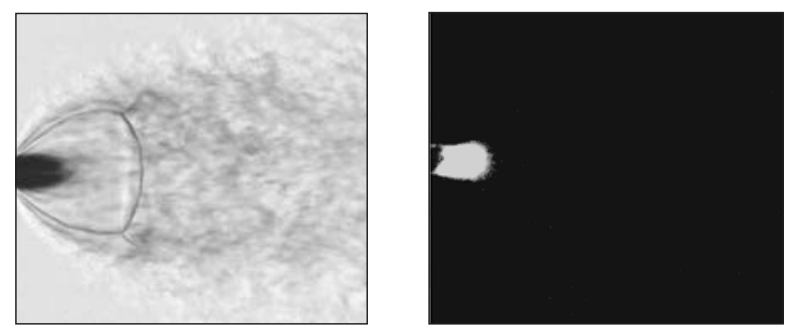

(b)

Figure 19 Supercritical $n$-hexane jets at different injection temperatures: $(a) T_{r}$ $=1.03$; and $(b) T_{r}=1.09$. Test conditions: $p_{\mathrm{inj}} / p_{c}=6.75, T_{\mathrm{chm}}=957 \mathrm{~K}, p_{\mathrm{chm}}=5 \mathrm{bar}$, and $p_{r}=0.16$

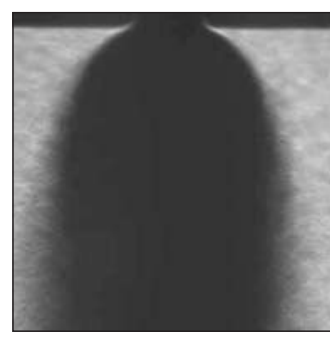

(a)

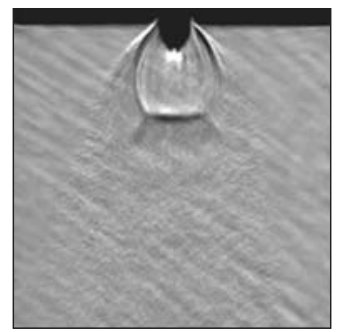

(b)

Figure 20 Supercritical methane/ethylene jets at different injection temperatures: (a) $T_{r}=1.03$; and (b) $T_{r}=1.23$. Test conditions: $x_{\mathrm{CH}_{4}}=0.1, p_{\text {inj }} / p_{c}=1.15$, $T_{\mathrm{chm}}=300 \mathrm{~K}, p_{\mathrm{chm}}=0.17 \mathrm{MPa}$, and $p_{r}=0.03$. Courtesy of $[40]$

near the nozzle exit is similar to that observed in a highly underexpanded idealgas jet. As the injection temperature approaches the critical temperature (i. e., $\left.T_{r}=1.03\right)$, keeping constant all other parameters, the supercritical spray experiences a gas-like expansion process with a discernible, continuous liquid core. The plume appears extremely opaque in the shadowgraph image. According to the 
observations of $[41,42]$ for supercritical ethylene sprays, the opaque appearance of the spray derives from condensation of the methane/ethylene mixture when the supercritical temperature is approached. The condensation in the $T_{r}=1.03$ jet appears immediately at the nozzle exit, indicating that the condensation may occur right at the nozzle exit or even inside the injector.

A similar phenomenon has been observed also in the authors' shock tube experiments. Figure 19 compares the spray structure of two supercritical sprays with an initial fuel temperature of $522 \mathrm{~K}\left(T_{r}=1.03\right)$ and $554 \mathrm{~K}\left(T_{r}=1.09\right)$, respectively, and a back pressure of $p_{\mathrm{chm}}=5$ bar. The presence of a liquid phase is proven by means of Mie scattering images. As can be seen, the spray behavior resembles that of a gaseous jet for $T_{r}=1.09$ with hardly any liquid region within the spray. For $T_{r}=1.03$, the overlapping Mie image shows the existence of a distinct liquid area within the gaseous jet due to the onset of condensation within the nozzle.

The formation of the liquid phase occurs through a highly nonequilibrium process. The inception of condensation can be schematically described as follows. During its expansion through the nozzle, the gaseous phase may depart drastically from the equilibrium phase distribution. A state of supersaturation (e.g., $\Phi$ $\left.=p_{v} / p_{\text {sat }}>1\right)$ is therefore achieved, indicative of a metastable nonequilibrium state of the vapour phase. Here, $p_{v}$ is the vapour pressure and $p_{\text {sat }}$ is the equilibrium vapour pressure at the corresponding local temperature. The actual phase transition is preceded by homogeneous (and/or heterogeneous) nucleation. Homogeneous nucleation refers to the spontaneous formation, within the vapour phase, of stable clusters by the kinetic process of detachment and impingement of molecules. As these metastable clusters exceed some critical size, the process of droplet growth sets in, leading to the formation of a stable liquid phase. The accompanying release of latent heat to the flow has a strong impact on the temperature and pressure distribution within the expanding fluid and may even lead to a chocked flow in the nozzle [43].

As corroborated by experimental evidence, the inception of nonequilibrium condensation is more likely to occur in fluids with an injection temperature close to the critical value (e.g., $T_{r}=1.03$ ). This is due to two concomitant factors. First, if the injection temperature is slightly above the critical value, the supercritical fluid is more likely to enter the two-phase region during its (isentropic) expansion within the nozzle, even for very low expansion ratios (e.g., $\left.p_{\text {inj }} / p_{\text {chm }} \approx 1.5\right)$. Second, the probability of inception of homogeneous condensation increases considerably for a near-critical fluid, even for a relatively low supersaturation $\Phi=O(1)$. This is due to the fact that the onset of nucleation (i.e., the creation of a stable cluster population for which it is energetically favourable to grow) requires the exceedance of the energy (activation) barrier for generating each critical embryo. This activation barrier is strongly dependent on supersaturation and surface tension [5]. Since the surface tension tends to zero as the fluid approaches its critical point, this implies that, for a given 


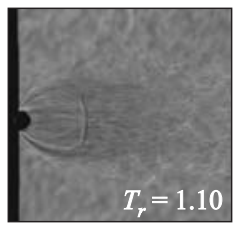

(a)

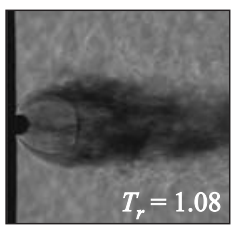

(b)

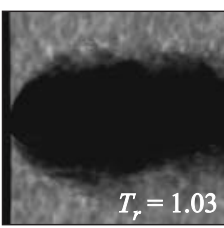

(c)

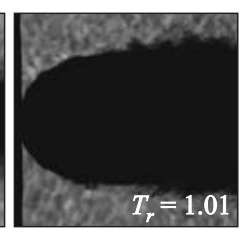

(d)

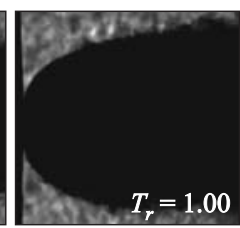

(e)

Figure 21 Effect of varying the fuel temperature on the injection of a supercritical fuel: (a) $T_{\mathrm{inj}}=311 \mathrm{~K}\left(T_{r}=1.10\right) ;(b) 300$ (1.08); (c) 295 (1.03); (d) 285 (1.01); and (e) $T_{\mathrm{inj}}=281 \mathrm{~K}\left(T_{r}=1.00\right)$. Test conditions: $p_{\text {inj }}=5.14 \mathrm{MPa}, p_{\mathrm{chm}}=0.14 \mathrm{MPa}$, $T_{\mathrm{chm}}=300 \mathrm{~K}$, and $p_{r}=0.03$. Fuel: ethylene. Courtesy of [44]

supersaturation, a near-critical fluid will have both an infinitesimally small activation barrier $\Delta G_{n}$ and critical embryo size $r^{*}$. A second implication is that, for a given supersaturation, higher nucleation rates have to be expected for a nearcritical fluid, as it is highly probable that a large number of embryos will be able to surmount the infinitesimally small energy barrier. In [40], it is estimated nucleation rates of the order of $10^{37} \mathrm{~m}^{-3} \mathrm{~s}^{-1}$ at $\Phi \leq 2$, while in steam turbine applications nucleation rates of the order of $10^{24} \mathrm{~m}^{-3} \mathrm{~s}^{-1}$ at $\Phi \geq 25$ are typically encountered. This explains why the formation of condensate is strongly dependent on temperature and it is most likely to occur for reduced temperatures near one as confirmed by the experimental observations of the authors of $[40,42]$.

Hereafter, the visual characteristics of a supercritical jet are discussed. The analysis presented here is still mostly at a phenomenological level and focuses with particular emphasis on the differences between a gaseous and a nucleating supercritical jet. Figure 21 shows the effect of varying the injection temperature. These experiments were performed by Lin et al. [40] and show an ethylene jet injected in a 0.14-megapascal nitrogen environment for injection temperatures of $311,300,295,285$, and $281 \mathrm{~K}$, corresponding to reduced temperatures of 1.10 , $1.08,1.03,1.01$, and 1.00 , respectively. As the injection temperature approaches the temperature of the critical point, the ethylene jets show a long fuel core caused by fuel condensation. As $T_{\text {inj }}$ increases, condensate formation is less significant and the Mach disk structure becomes visible. The dome-shape of the jet at the injector exit suggests that a Mach disk exists always, although it is not visible. The presence of the Mach disk, in fact, essentially depends upon the expansion ratio $p_{\text {inj }} / p_{\mathrm{chm}}$ and occurs only in the case of underexpanded jets. Note that for a condensing underexpanded jet, the flow pattern appears to be far more complicated, exhibiting both single and two-phase regions. In fact, the fluid along streamlines passing through the Mach disk is less likely to condense, due to the substantial increases in pressure and temperature after the Mach disk. The fluid along streamlines passing through the barrel shock is, instead, more likely to stay in the two-phase region after the barrel shock. 


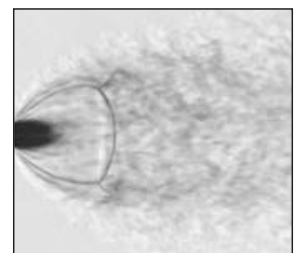

(a)

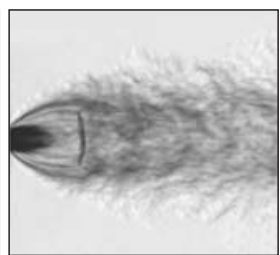

(b)

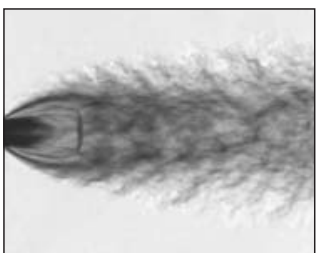

(c)

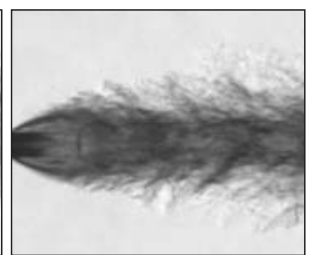

$(d)$

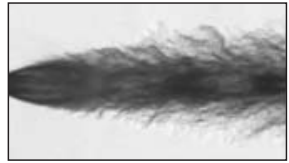

(e)

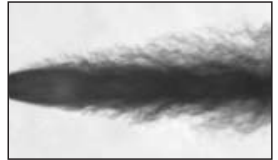

$(f)$

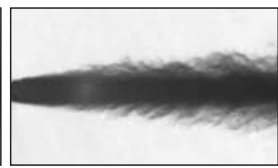

$(g)$

Figure 22 Effect of varying the ambient pressure on the injection of a supercritical fuel: (a) 5 bar $\left(P_{r}=0.16\right)$; (b) 10 (0.33); (c) 15 (0.49); (d) 20 (0.66); (e) 27.5 (0.91); $(f) 35(1.15)$; and $(g) 42.5$ bar $\left(P_{r}=1.40\right)$. Test conditions: $p_{\text {inj }}=200$ bar, $T_{\mathrm{inj}}=554 \mathrm{~K}$, and $T_{\mathrm{chm}}=957 \mathrm{~K}\left(T_{r}=1.09\right)$. Fuel: $n$-hexane

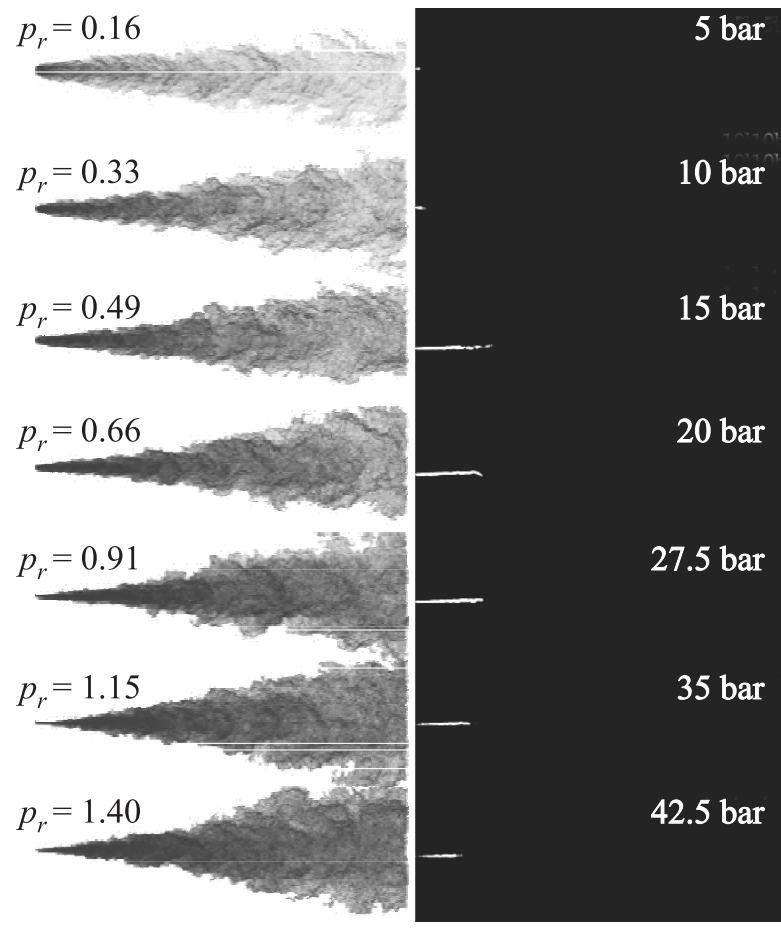

Figure 23 Comparison of Schlieren and Mie scattering images for a supercritical spray and varying back pressures. Test conditions: $p_{\text {inj }}=200 \mathrm{bar}, T_{\mathrm{inj}}=522 \mathrm{~K}$, and $T_{\mathrm{chm}}=957 \mathrm{~K}\left(T_{r}=1.09\right)$. Fuel: $n$-hexane 
Figure 22 illustrates the effects of varying the ambient pressure on the jet morphology. For high expansion ratios (i.e., $p_{\text {inj }} / p_{\text {chm }} \geq 15$ ), the dome-shaped spray boundary, necessary to accommodate the larger pressure drop, is clearly visible. This bowl shape of the spray becomes less pronounced when the chamber pressure is increased.

Several authors [40, 42] reported a similitude with an ideal-gas jet with respect to the location of the Mach disk $x_{\mathrm{M}}$ : the measured $x_{\mathrm{M}}$ positions, in fact, match very well the correlation developed in [45] for an ideal-gas jet. These findings confirm the fact that the axial location of the Mach disk is primarily a function of the expansion ratio $p_{\mathrm{inj}} / p_{\mathrm{chm}}$ and is independent of fuel type and fuel condensation. Note that the similitude to an ideal-gas jet is really limited only to the position of the Mach disk. In fact, the comparison between Schlieren and Mie scattering images, shown in Fig. 23, reveals the presence of a liquid core in all cases. In agreement with the experimental observation of [40], the fluid passing through the Mach disk is less likely to condense, as corroborated by the short axial extension of the liquid core for the cases $p_{\mathrm{chm}}=5$ and $10 \mathrm{bar}$ in Fig. 23. Fuel condensation has, instead, a direct impact on the size of the Mach disk $D_{\mathrm{M}}$. The latter is larger compared to a gaseous jet to compensate for the increase in static pressure due to the phase transition process. Several authors (see, e.g., $[40,42,45])$ confirmed this trend and reported an increase in the diameter of the Mach disk with increasing expansion ratio $p_{\text {inj }} / p_{\text {chm }}$.

Figure 24 shows a magnified

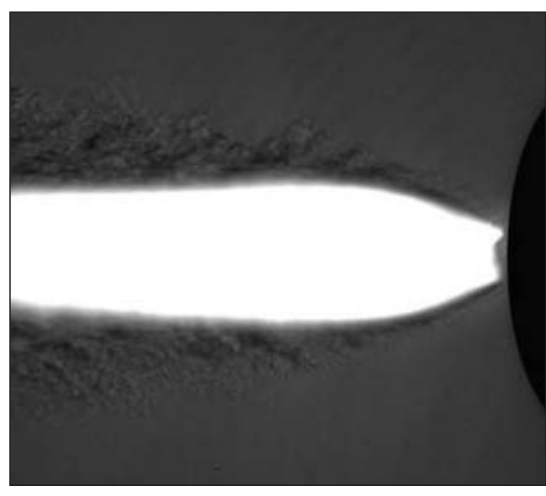

Figure 24 Superimposed shadowgraph and Mie signal in a supercritical jet. Test conditions: $T_{f}=554 \mathrm{~K}, T_{\mathrm{chm}}=957 \mathrm{~K}, p_{\mathrm{chm}}=20$ bar. Fuel: $n$-hexane evident that the fuel exiting the nozzle is still in a supercritical state, due to the absence of any Mie signal in this region. This represents the first experimental evidence that the onset of nonequilibrium condensation occurs in the jet plume, 
as a consequence of the rapid cooling experienced by the flow through the two expansion fans, positioned at the nozzle exit in an underexpanded nozzle flow.

\section{CONCLUDING REMARKS}

A double-diaphragm shock tube has been designed for fluid disintegration studies at near-critical conditions. Since the occurrence of different disintegration regimes (e.g., transcritical and supercritical) depends strongly upon the ambient and injection conditions, considerable emphasis is placed on the reproducibility and uniformity of the test conditions. For this purpose, the facility has been upgraded with a variable-area driver section to compensate for shock attenuation and boundary layer effects. Shock tube qualification tests clearly show that a good spatial and temporal uniformity of test conditions can be attained and only slight fluctuations in pressure $(<2 \%)$ are detected. Furthermore, the injection of preheated fuel at near-critical conditions can be successfully performed with a high degree of reproducibility.

To demonstrate the feasibility of near-critical jet disintegration studies in the DDST, two experimental campaigns have been performed in the transcritical and supercritical regime, respectively. Based on the shock tube experiments, the main features of each disintegration regime have been discussed and the authors' findings have been compared to the literature data to verify their consistency and reliability.

The transcritical breakup mode encompasses the transitions a liquid (at subcritical temperature and supercritical pressure) may undergo when injected into an environment at supercritical conditions. If the initial fluid temperature is considerably lower than the critical temperature (i. e., $T_{r} \ll 1$ ), the liquid fragmentation resembles fully the atomization regime and the spray angle increases linearly with chamber pressure. For injection temperatures approaching the critical temperature (i. e., $T_{r} \geq 0.8$ ), two distinct regions can be identified: a downstream and a near-nozzle region. In the downstream region $(x / D \geq 22)$, analysis of Mie and Schlieren images reveals a single-phase gaseous jet appearance, dominated by turbulent shear-layer mixing effects. In the near-nozzle region (up to $x / D \approx 22$ ), experimental evidences indicate a two-phase spray structure. The spray is composed of an inner liquid core surrounded by a shear transitional mixing layer. The spray angle is only weakly dependent upon the chamber pressure and in the limit (i. e., $p_{r} \gg 1$ ) it exhibits a functional dependence similar to that of a compressible gaseous mixing layer.

The disintegration of a supercritical spray resembles an ideal-gas expansion at injection conditions away from the thermodynamic critical point. If the fluid injection temperature approaches the critical value, droplet condensation, initiated by homogeneous nucleation, occurs downstream of the nozzle exit plane and creates an opaque spray appearance in shadowgraph images. At near-critical 
condition, the inception of a liquid phase is favoured by the drastic reduction in surface tension, which results in an infinitesimally small activation barrier and critical embryo size. The onset of condensation has a strong impact on the spray visual characteristics. First, the spray exhibits a long liquid core and loses its similarity to a gaseous spray. Second, for underexpanded sprays, a more complicated pattern is observed. The fluid along streamlines passing through the Mach disk is less likely to condense, due to the relatively short residence time within the two-phase and liquid regions and substantial increases in pressure and temperature after the Mach disk. The fluid along streamlines passing through the barrel shock is more likely to stay in the two-phase region after the barrel shock. The location of the Mach disks, instead, is only a function of the expansion ratio and is independent of fuel type and condensation.

In conclusion, the shock tube data not only corroborated the available literature database, but shed also additional light on the internal structure of a near-critical spray, thus proving the well-foundness of Bellan's theoretical analysis $[2,32]$.

\section{ACKNOWLEDGMENTS}

This work was performed within the Long-Term Advanced Propulsion Concepts and Technologies (LAPCAT) project investigating high-speed air-breathing propulsion. LAPCAT, coordinated by ESA-ESTEC, is supported by the EU within the 6th Framework Programme Priority 1.4, Aeronautic and Space, Contract No. AST4-CT-2005-012282. Further information on LAPCAT can be found on: http://www.esa.int/techresources/lapcat.

The authors are also gratefully indebted to the Landesstiftung BadenWürttemberg for facilitating the high-speed experiments entailed in this paper.

\section{REFERENCES}

1. Mayer, W., and J. Telaar. 2002. Investigation of breakup of turbulent cryogenic variable-density jets. Atomization Sprays 12(5-6):651-66.

2. OkongTo, N. A., and J. Bellan. 2002. Direct numerical simulation of a transitional supercritical binary mixing layer: Heptane and nitrogen. J. Fluid Mech. 464:1-34.

3. Zong, N., H. Meng, S.-Y. Hsieh, and V. Yang. 2004. A numerical study of cryogenic fluid injection and mixing under supercritical conditions. Phys. Fluids 16(12):424861.

4. Oschwald, M., J. J. Smith, R. Branam, J. Hussong, A. Schik, B. Chehroudi, and D. Talley. 2006. Injection of fluids into supercritical environments. Combust. Sci. Technol. 178:49-100.

5. Lamanna, G., I. Stotz, and B. Weigand. 2009. Supercritical fluid injection in quiescent flow fields: Modelling and mixing enhancement. In: Liquid fragmentation 
in high speed flows. VKI lecture ser. Rhode-Saint-Genese, Belgium: Von Karman Institute for Fluid Dynamics. Vol. VKI-LS-2009-04.

6. Hermanson, J., P. Papas, and I. Kay. 1994. Structure and penetration of a supercritical fluid jet in supersonic flow. J. Propul. Power 10(3):387-94.

7. Stotz, I., G. Lamanna, H. Hettrich, B. Weigand, and J. Steelant. 2008. Design of a double diaphragm shock tube for fluid disintegration studies. Review of Scientific Instruments 79(12):125106-1-125106-9.

8. Mirels, H. 1955. Laminar boundary layer behind shock advancing into stationary fluid. Technical Report TN 3401. NACA.

9. Rudinger, G. 1961. Effect of boundary-layer growth in a shock tube on shock reflection from a closed end. Phys. Fluids 4:1463-73.

10. Petersen, E. L., and R. K. Hanson. 2003. Improved turbulent boundary-layer model for shock tubes. AIAA J. 41(7):1314-22.

11. Petersen, E. L., and R.K. Hanson. 2001. Nonideal effects behind reflected shock waves in a high-pressure shock tube. Shock Waves 10:405-20.

12. Dumitrescu, L. Z. 1972. An attenuation-free shock tube. Phys. Fluids 15(1):207-09.

13. Stotz, I., G. Lamanna, B. Weigand, and J. Steelant. 2006. A double-diaphragm shock tube for hydrocarbon disintegration studies. AIAA Paper No. 2006-8109.

14. Jacobs, P. A. 1994. Quasi-one-dimensional modelling of free-piston shock tunnels. AIAA J. 32(1):137-45.

15. Jacobs, P. A. 1998. Shock tube modelling with L1d. Technical report. Department of Mechanical Engineering. The University of Queensland.

16. Schardin, H. 1955. Über das stosswellenrohr. Technical Report $14 \mathrm{~m} / 51$. Laboratoire de Recherches de Saint-Louis (LRSL). 231-52.

17. Schardin, H. 1958. Ein Beispiel zur Verwendung des Stoßwellenrohres für Probleme der instationären Gasdynamik. Zeitschrift für Angewandte Mathematik und Physik (ZAMP) 9(5-6):606-21.

18. Hong, Z., G. A. Pang, S.S. Vasu, D.F. Davidson, and R. K. Hanson. 2009. The use of driver inserts to reduce non-ideal pressure variations behind reflected shock waves. Shock Waves 19(2):113-23.

19. Chang, A. Y., E. C. J. Rea, and R. K. Hanson. 1987. Temperature measurements in shock tubes using a laser-based absorption technique. Appl. Optics 26(5):885-91.

20. Oehlschlaeger, M. A., D. F. Davidson, and J. B. Jeffries. 2005. Temperature measurement using ultraviolet laser absorption of carbon dioxide behind shock waves. Appl. Optics 44(31):6599-605.

21. Davidson, D. F., R. W. Bates, E. L. Petersen, and R. K. Hanson. 1998. Shock tube measurements of the equation of state of argon. Int. J. Thermophys. 19(6):1585-94.

22. Otsu, N. 1979. A threshold selection method from gray-level histograms. IEEE Trans. Systems Man Cybernetics 9(1):62-66.

23. Lefebvre, A. H. 1989. Atomization and sprays. Taylor \& Francis Inc.

24. Naber, J. D., and D.L. Siebers. 1996. Effects of gas density and vaporization on penetration and dispersion of diesel sprays. SAE International Congress and Exposition. SAE Paper No. 960034.

25. Kang, J., C. Bae, and K. O. Lee. 2003. Initial development of non-evaporating diesel sprays in common-rail injection systems. Int. J. Engine Res. 4:283-98. 
26. Klein-Douwel, R.J.H., P. J. M. Frijters, L.M. T. Somers, W. A. de Boer, and R.S. G. Beart. 2007. Macroscopic diesel fuel spray shadowgraphy using high speed digital imaging in a high pressure cell. Fuel 86(12-13):1994-2007.

27. Siebers, D. L. 1998. Liquid-phase fuel penetration in diesel sprays. Society of Automotive Engineers Technical Papers. 1205-27. Paper No. 980809.

28. Adam, A., P. Leick, G. Bittlinger, and C. Schulz. 2009. Visualization of the evaporation of a diesel spray using combined Mie and Rayleigh scattering techniques. Exp. Fluids 47:439-99.

29. Newman, J. A., and X. Brzustowski. 1971. Behavior of a liquid jet near the thermodynamic critical region. AIAA J. 9:1595-99.

30. Mayer, W., J. Telaar, R. Branam, G. Schneider, and J. Hussong. 2001. Characterization of cryogenic injection at supercritical pressure. AIAA Paper No. 2001-3275.

31. Chehroudi, B., D. Talley, and E. Coy. 2002. Visual characteristics and initial growth rates of round cryogenic jets at subcritical and supercritical pressures. Phys. Fluids 14(2):850-61.

32. Bellan, J. 2000. Supercritical (and subcritical) fluid behaviour and modeling: Drops, streams, shear and mixing layers, jets and sprays. Prog. Energy Combust. Sci. 26:329-66.

33. Harstad, K., and J. Bellan. 2000. An all-pressure fluid drop model applied to a binary mixture: Heptane in nitrogen. Int. J. Multiphase Flow 26:1675-706.

34. Abramovich, G. N. 1963. The theory of turbulent jets. M.I.T. Press.

35. Hiroyasu, H., and M. Arai. 1990. Structures of fuel sprays in diesel engines. Doc. No. 900475.

36. Atsavapranee, P., and M. Gharib. 1997. Structures in stratified plane mixing layers and the effects of cross-shear. J. Fluid Mech. 342:53-86.

37. Dimotakis, P. E. 1986. Two-dimensional shear-layer entrainment. AIAA J. 21:179196.

38. Papamoschou, D., and A. Roshko. 1988. The compressible turbulent shear layer: An experimental study. J. Fluid Mech. 197:453-77.

39. Zong, N., and V. Yang. 2006. Cryogenic fluids jets and mixing layers in transcritical and supercritical environments. Proc. Combust. Inst. 178:193-227.

40. Lin, K.-C., S. K. Cox Stouffer, and T. A. Jackson. 2006. Structures and phase transition processes of supercritical methane/ethylene mixtures injected into a subcritical environment. Combust. Sci. Technol. 178:129-60.

41. Wu, P.-K., T. H. Chen, A. S. Nejad, and C. D. Carter. 1996. Injection of supercritical ethylene in nitrogen. J. Propul. Power 12:770-77.

42. Wu, P., M. Shahnam, K. Kirkendall, C. Carter, and A. S. Nejad. 1999. Expansion and mixing processes of underexpanded supercritical fuel jets injected into superheated conditions. J. Propul. Power 15(5):642-49.

43. Lamanna, G. 2000. On nucleation and droplet growth in condensing nozzle flows. Ph.D. Thesis. Technische Universiteit Eindhoven.

44. Star, A. M., J.R. Edwards, K. C. Lin, S. Cox-Stouffer, and T. A. Jackson. 2006. Numerical simulation of injection of supercritical ethylene into nitrogen. J. Propul. Power 22(4):809-19.

45. Crist, S., P. Sherman, and D. Glass. 1966. Study of the highly underexpanded sonic jet. AIAA J. 5:68-71. 